ARTICLE

https://doi.org/10.1038/s41467-019-13210-5

\title{
Rpd3L and Hda1 histone deacetylases facilitate repair of broken forks by promoting sister chromatid cohesion
}

\author{
Pedro Ortega1, Belén Gómez-González (iD) ${ }^{1 \star} \&$ Andrés Aguilera (i) ${ }^{1 \star}$
}

Genome stability involves accurate replication and DNA repair. Broken replication forks, such as those encountering a nick, lead to double strand breaks (DSBs), which are preferentially repaired by sister-chromatid recombination (SCR). To decipher the role of chromatin in eukaryotic DSB repair, here we analyze a collection of yeast chromatin-modifying mutants using a previously developed system for the molecular analysis of repair of replication-born DSBs by SCR based on a mini-HO site. We confirm the candidates through FLP-based systems based on a mutated version of the FLP flipase that causes nicks on either the leading or lagging DNA strands. We demonstrate that Rpd3L and Hda1 histone deacetylase (HDAC) complexes contribute to the repair of replication-born DSBs by facilitating cohesin loading, with no effect on other types of homology-dependent repair, thus preventing genome instability. We conclude that histone deacetylation favors general sister chromatid cohesion as a necessary step in SCR.

\footnotetext{
${ }^{1}$ Centro Andaluz de Biología Molecular y Medicina Regenerativa (CABIMER), Universidad de Sevilla-CSIC-Universidad Pablo de Olavide, Seville, Spain. *email: gomezb@us.es; aguilo@us.es
} 
T he maintainance of genetic information through cell divisions requires accurate duplication of the entire genome, despite the presence of DNA lesions ${ }^{1}$. Failures during DNA replication can lead to genome instability, most cancer mutations being attributed to replication errors ${ }^{2}$. The most common DNA lesions are single-strand DNA (ssDNA) breaks or nicks ${ }^{3,4}$, which when encountered by a replication fork are converted into double-strand breaks (DSBs) that are among the most harmful lesions. Besides nicks, fork stalling at other DNA lesions can also ultimately lead to fork collapse or even DSBs, particularly in the absence of a proper checkpoint-mediated fork stabilization ${ }^{5}$.

DSB repair can be accomplished by non-homologous end joining (NHEJ) or homologous recombination (HR) but replication-born DSBs are preferentially repaired by sister chromatid recombination (SCR), an HR reaction with the intact sister chromatid $^{6-8}$. Alternatively, replication-born DSBs can be repaired by break-induced replication (BIR) or by HR with other homologous templates such as ectopic sequences or the homologous chromosome with the risks of causing a plethora of genetic instability phenotypes including loss of heterozygosity, deletions, insertions, translocations, or gross chromosomal rearrangements $(\mathrm{GCRs})^{9}$. To favor SCR, sister chromatids are hold together by the cohesion ring ${ }^{10}$, which is formed by two SMC (structural maintenance of chromosomes) components (Smc1 and Smc3) and two non-SMC components (Sccl and Scc3). Sister chromatid cohesion at centromeres is essential and apparently sufficient for proper chromosome segregation in mitosis, but arm cohesion seems to have evolved to promote accurate post-replicative repair ${ }^{11}$. Indeed, although general cohesion is established after DNA replication, DNA damage triggers de novo cohesin loading facilitating repair ${ }^{12-15}$. The DNA damage response (DDR) promotes the establishment of such damage-induced cohesion at the break site as well as at other genomic regions independently of replication ${ }^{15-17}$. Damage-induced cohesion promotes SCR and is favored by certain factors such as the Smc5/6 complex ${ }^{18,19}$, the constitutive methylation of H3K79 (ref. ${ }^{20}$ ), and by chromatin remodeling by the RSC complex ${ }^{21}$.

In addition to cohesin proteins, several other factors have been described to contribute to the efficiency of SCR, such as the general HR factors Rad51, Rad52, Rad54, Rad59, Sae2, Sgs1, and Mus81, or the helicase Rrm3, which is part of the replisome ${ }^{22-24}$. The chromatin context at which the DSB occurs as well as the DSB-induced changes in chromatin can act as specific regulators of SCR. Thus, the higher H3K56 acetylation of newly synthesized $\mathrm{DNA}^{25}$ seems to provide to sister chromatids a preference to be used as a substrate for replication-born DSBs repair versus nonsister templates ${ }^{26}$. However, the mechanism by which SCR is regulated versus other HR mechanisms is unknown.

We previously developed a yeast TINV-HO plasmid system based on a 24-bp mini-HO site (HOr) to induce ssDNA breaks that resulted in DSBs after replication and permitted the molecular analysis of their repair by $\mathrm{SCR}^{6,10}$. Since that system caused nicks on either DNA strand of the HOr site, we developed a system carrying the $F R T$ recombination site of the yeast $2 \mu \mathrm{m}$ circle and the inducible expression of a mutated form of the FLP endonuclease that permitted to induce strand-specific nicks at the $F R T$ site. Using both sets of systems we have performed a detailed analysis of the impact of different chromatin factors, including remodelers and modifiers, on SCR. We identified Rpd3L and Hda1 histone deacetylases (HDACs) as specific regulators of SCR that prevent genetic instability. Interestingly, we show that both HDACs contribute to SCR by facilitating cohesin loading and promoting sister chromatid cohesion. These data allow us to propose a role for Hda1 and Rpd3L-mediated histone deacetylation in the maintenance of genome integrity by supporting cohesion, thus favoring the repair of replication-born DSBs by SCR over other forms of DSB repair.

\section{Results}

Identification of chromatin factors affecting SCR. To investigate the putative role of chromatin factors in the repair of replication-born DSBs, we analyzed unequal sister chromatid exchange (SCE), which is an accurate indicator of total SCR ${ }^{6,10}$. We built a plasmid containing both the previously reported TINV-HO system and the HO endonuclease gene under the control of the GAL1 galactose-inducible promoter and examined a selection of 27 mutants in chromatin remodelers and histone modifiers from the Euroscarf mutant collection. The TINV-HO system is based on two leu2 inverted repeats, one of which contains the HOr site, which is inefficiently targeted by the endonuclease $\mathrm{HO}$ leading mainly to DNA nicks that are converted into DSBs by the replication fork (Fig. 1a) ${ }^{6,10}$. DSB and SCE intermediates were detected by Southern blot analysis after wild type and mutant strains were induced to express the $\mathrm{HO}$ endonuclease (Fig. 1a, Supplementary Fig. 1a, see Methods). The quantification of the 4.7-kb SCE-specific band confirmed a 2-4-fold decrease in SCE levels in four control mutants, previously reported to be affected in SCR $(\operatorname{dot} 1 \Delta, \operatorname{rad} 54 \Delta, r s c 1 \Delta$, and $r s c 2 \Delta)$. Five other

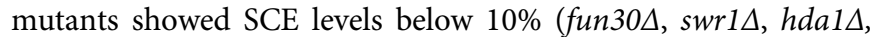
$r p d 3 \Delta$, and sap30 $)$ whereas $r p h 1 \Delta$ could not be analyzed due to its growth defect ${ }^{27}$ (Fig. 1b). Among them, Sap30 and Rpd3 belong to the Rpd3L HDAC complex. Rpd3 is a class I HDAC that acts as the catalytic subunit and can also be part of another HDAC complex, Rpd3S. Both Rpd3L and Rpd3S complexes share the Sin 3 and Ume1 subunits in addition to Rpd 3 while they also contain specific subunits such as Sap30 or Rco1, which are specific for Rpd3L and Rpd3S, respectively ${ }^{28}$. We therefore added $\operatorname{sap} 30 \Delta, r \operatorname{co} 1 \Delta$, and $\sin 3 \Delta$ for further analysis.

We next performed the same SCE analysis in a W303 genetic background (isogenic WS strains), which carries a deletion of the endogenous LEU2 gene to avoid interference with the leu2 repeats, and in which the $\mathrm{HO}$ endonuclease was expressed from the chromosome. The quantification of recombination intermediates after 3,6 , and $9 \mathrm{~h}$ of $\mathrm{HO}$ induction revealed that four of the mutants (fun $30 \Delta, h d a 1 \Delta, \sin 3 \Delta$, and $\operatorname{sap} 30 \Delta$ ) were strongly affected in the efficiency of SCE whereas swr1 $1 \Delta$ and $r p h 1 \Delta$ showed SCE levels similar to the wild type (Fig. 1c). Importantly, the percentage of HO-induced DSB was not significantly affected in any of the mutants but in sap30 , which grew more slowly and presented a delayed DSB induction with respect to the wild type (Supplementary Fig. 1b).

The TINV-HO system also allows studying genetically the appearance of $\mathrm{Leu}^{+}$recombinants, which appear mostly by SCR, but that can be the consequence of other HR events. In agreement with a defect in SCR, $r s c 2 \Delta$, hda1 $\Delta, \sin 3 \Delta$, and $\operatorname{sap} 30 \Delta$ showed a significant decrease in the levels of $\mathrm{HO}$-induced recombination $(+\mathrm{HO})$ (Fig. 1d). We also detected decreased recombination in rph1 1 , suggesting that this mutant might be also affected in some other HR type of events. However, fun30 $\Delta$ and swr1s efficiently repaired HO-induced breaks. The spontaneous $(-\mathrm{HO})$ levels of recombination in these mutants were either not affected or higher than in the wild type (Fig. 1d). Higher levels of spontaneous inverted-repeats recombination in swr $1 \Delta$ have been previously reported and are in agreement with a role for Swr1 in the maintenance of genetic stability ${ }^{29}$. By contrast, the increases observed in $r s c 2 \Delta$, $h d a 1 \Delta$, and sap $30 \Delta$ might reflect the incapacity of these strains to repair the replication-born DSBs via SCR (Fig. 1c), channeling spontaneous damage to other templates such as the other leu2 copy located in the same plasmid. Altogether, our screening results point to a specific role for Hda1 and Rpd3L, 
a

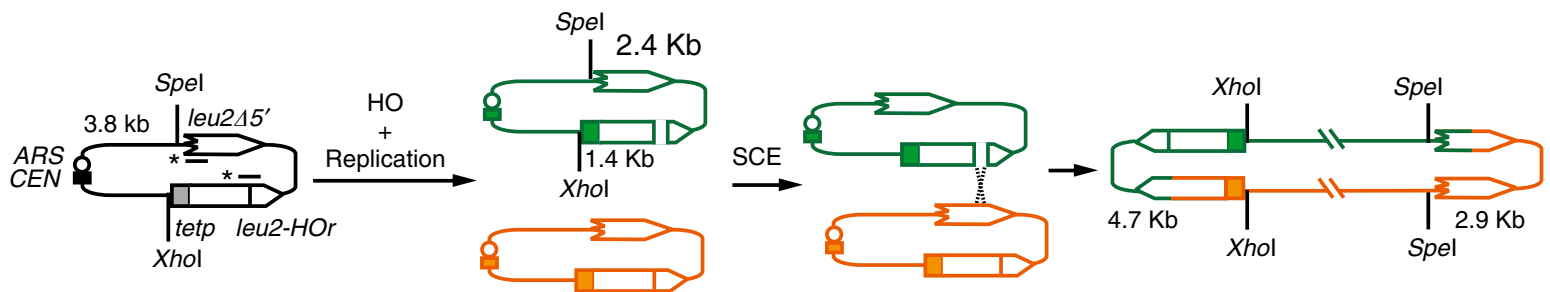

b

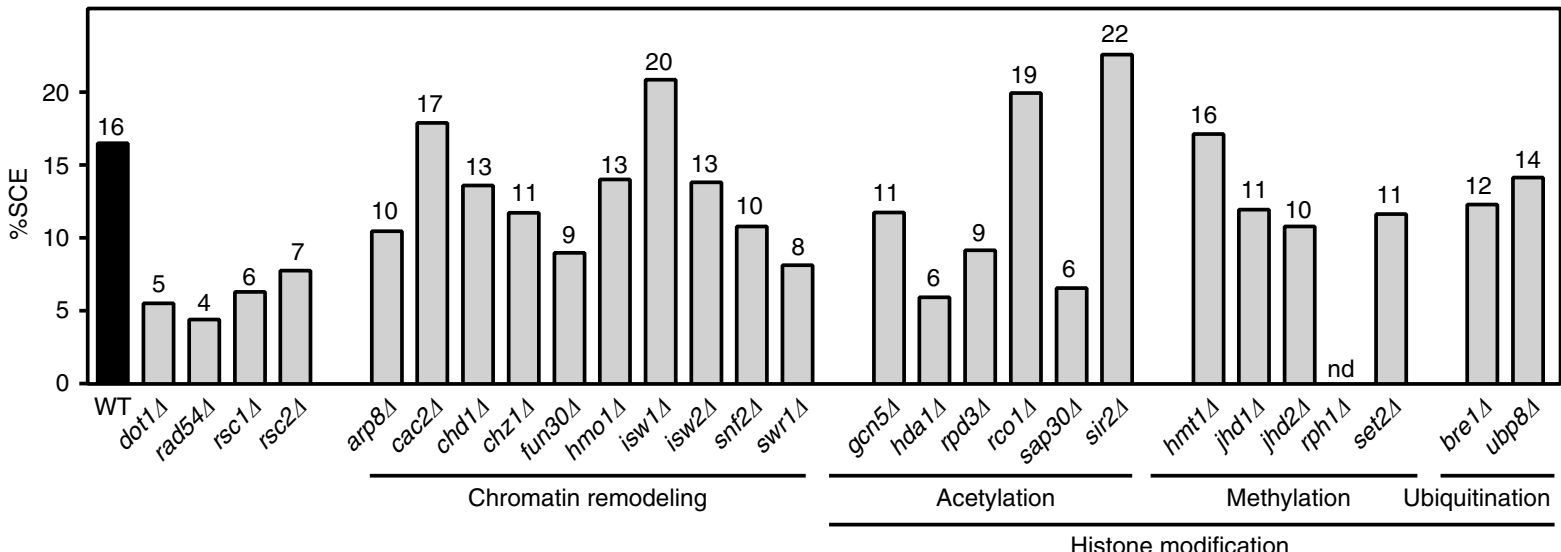

C
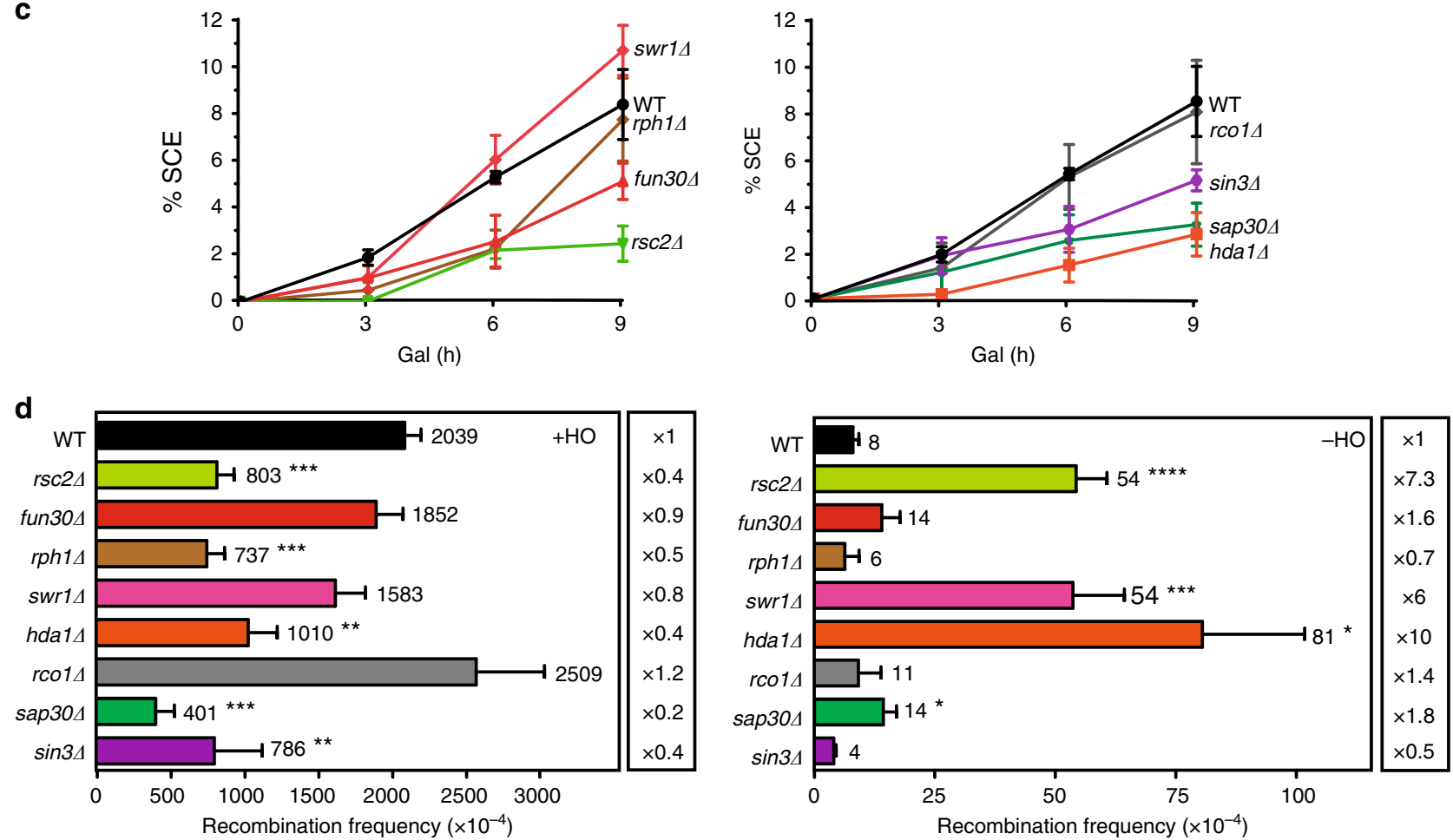

Fig. 1 Screening for chromatin factors involved in SCR. a Schemes of the TINV-HO system and SCE intermediates produced after HO-induced replicationborn DSBs. Fragments generated after Xhol-Spel digestion are indicated with their corresponding sizes in kb and were detected by Southern blot hybridization with a LEU2 probe (line with an asterisk). b Quantification of the SCE 4.7-kb fragment detected after $9 \mathrm{~h}$ of HO induction in wild type (BY4741) and the indicated mutant strains from the Euroscarf collection (see Supplementary Table 1) transformed with the pTHGH plasmid that contains both the TINV-HO system and GAL::HO $(n=1)$. c Quantification of the 4.7-kb SCE fragments during a time-course experiment after HO induction

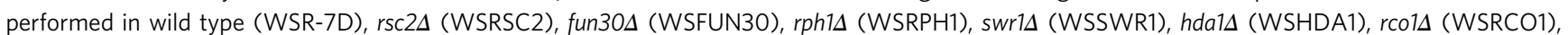
sap30 4 (WSSAP30), and $\sin 3 \Delta$ (WSSIN3) transformed with pRS316-TINV $(n \geq 2)$. d Analysis of spontaneous ( $-\mathrm{HO})$ and HO-induced (+HO)

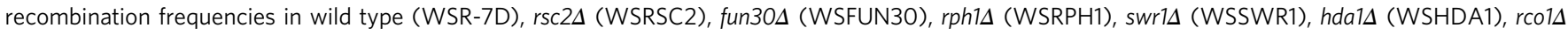
(WSRCO1), sap30 (WSSAP30), and $\sin 3 \Delta$ (WSSIN3) strains transformed with pRS316-TINV $(n \geq 3)$. Means and SEM are plotted in $\mathbf{c}$ and $\mathbf{d} .{ }^{*} p \leq 0.05$; ${ }^{\star \star} p \leq 0.01 ;{ }^{\star \star \star} p \leq 0.001 ;{ }^{\star \star \star \star} p \leq 0.0001$ (two-tailed Student's $t$-test). See also Supplementary Fig. 1. nd, not determined. Data underlying this figure are provided as Source Data file 
a
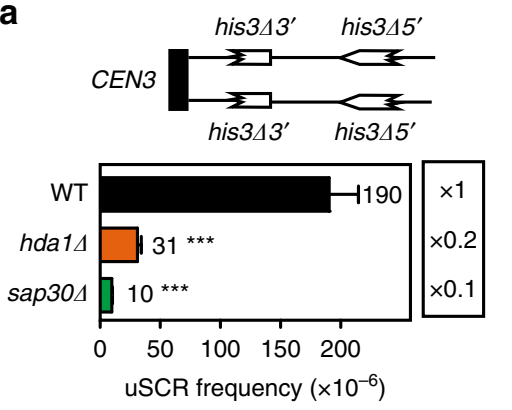

d

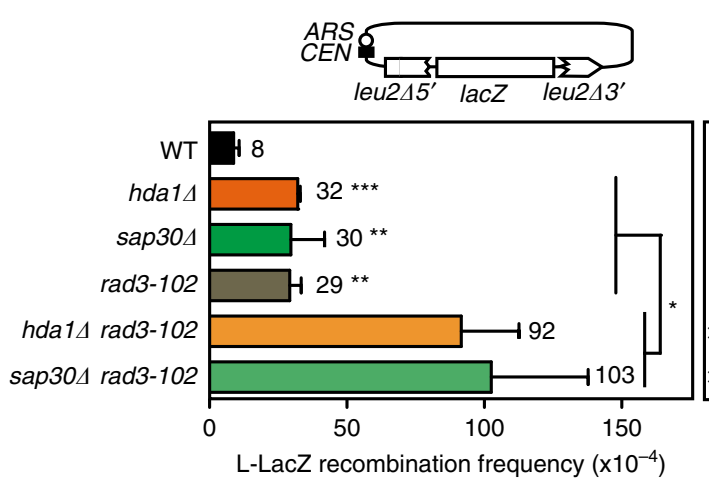

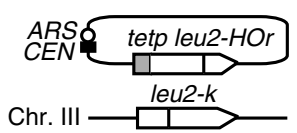

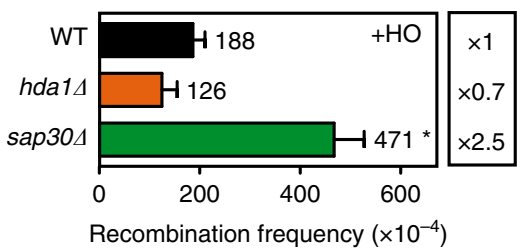

C
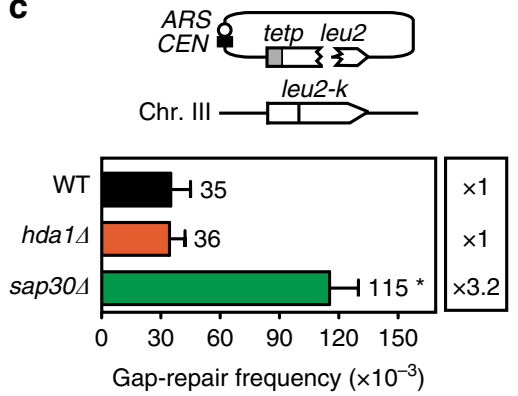

e

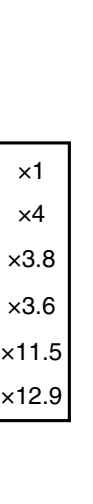

e

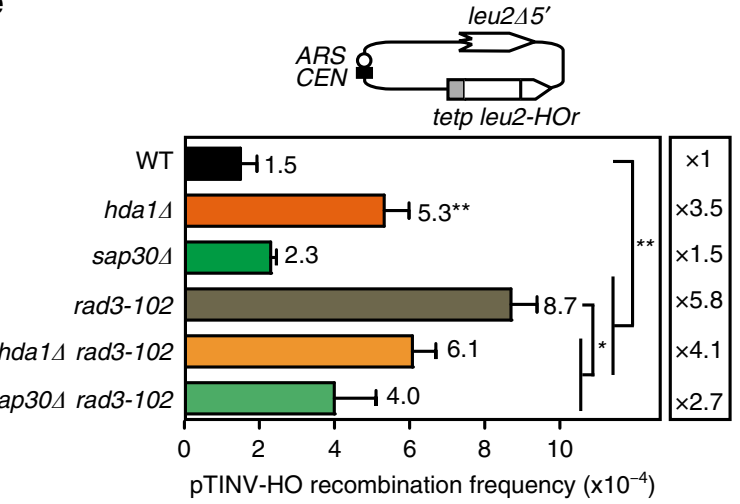

$\mathbf{f}$
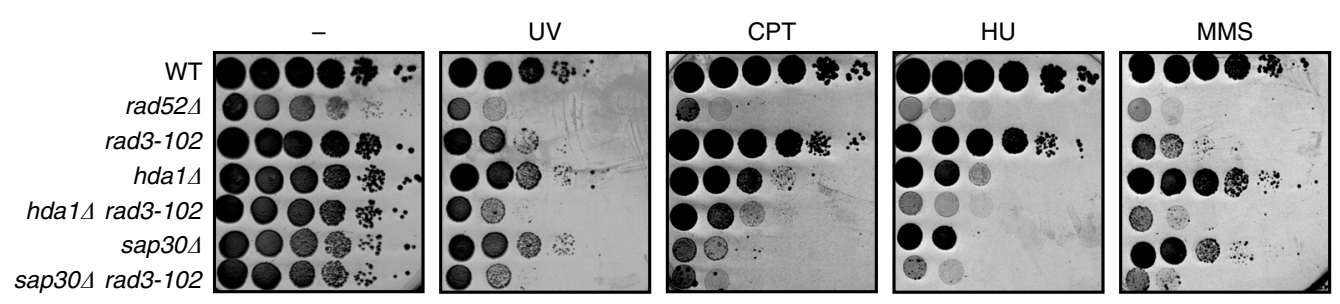

g
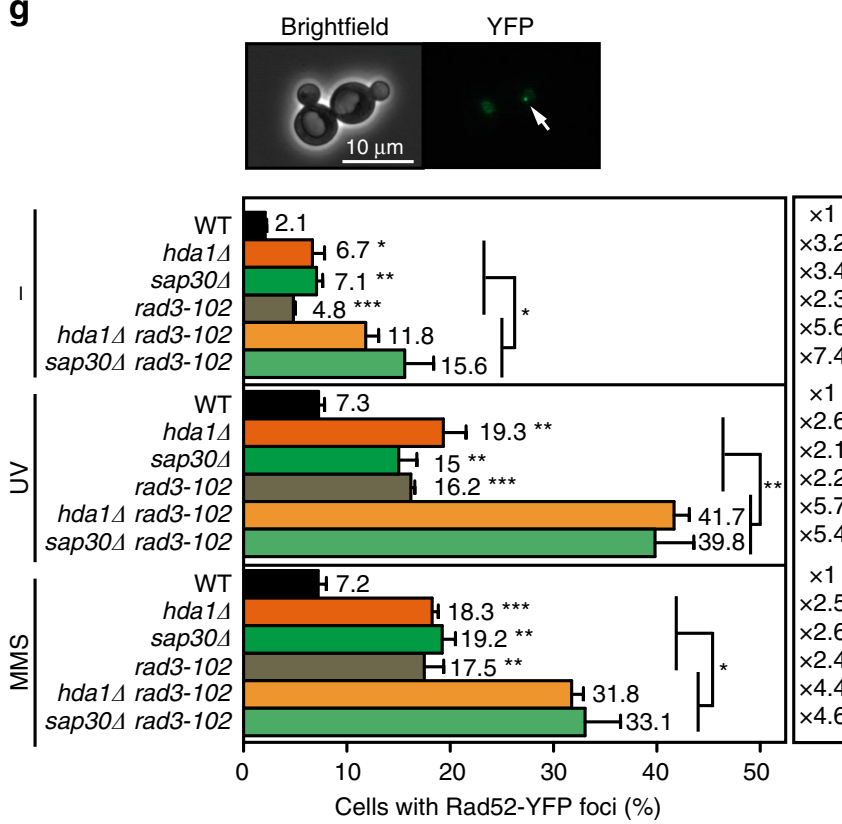

h

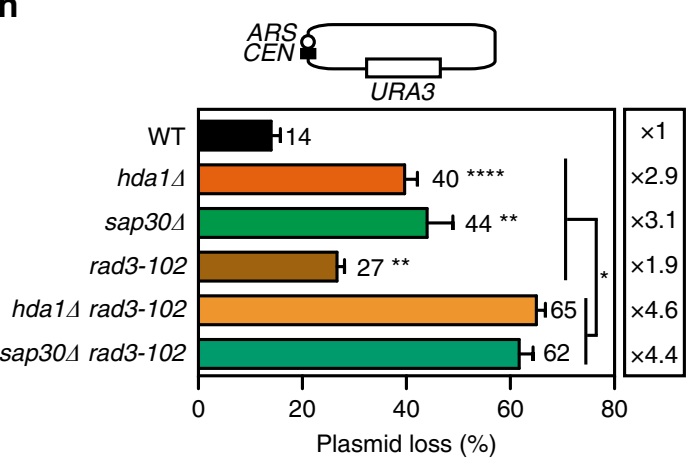

i
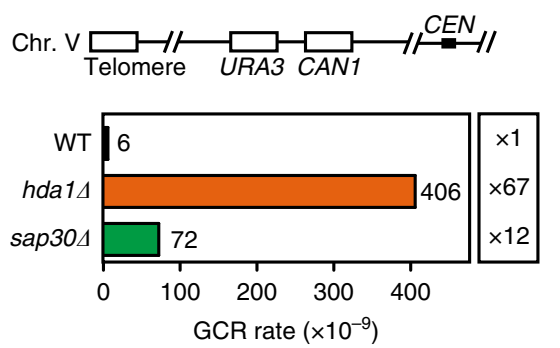

but not Rpd3S, in the repair of replication-born DSBs using the sister chromatid as a template.

Defective repair of replication-born DSBs in $h d a 1 \Delta$ and

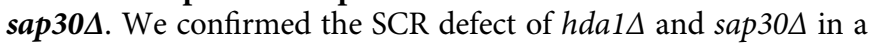

different system located in a chromosome that measures spontaneous unequal $\mathrm{SCR}^{30}$ (Fig. 2a) indicating that hdals and $\operatorname{sap} 30 \Delta$ affect the repair of not only HO-induced but also spontaneous DSBs, regardless of whether located in a plasmid or chromosome. By contrast, hda1s and sap30 showed no defect in the repair with non-sister templates, as shown for 
Fig. 2 Defective repair of replication-born DSBs in hda14 and sap304. a Analysis of spontaneous unequal SCR frequencies with the chromosomal directrepeat his3- $\Delta 5^{\prime}:$ :his3- $\Delta 3^{\prime}$ system in wild type (USCE), hda1s (USHDA1), and sap30 $\Delta$ (USSAP30) strains ( $n \geq 3$ ). b Analysis of HO-induced plasmid-chromosome recombination frequencies in wild type (W-Lk), hda14 (WLHDA1), and sap304 (WLSAP30) strains transformed with pCM189-

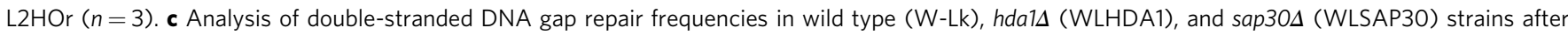
transformation with Mfel-digested pCM189-L2HOr or undigested pCM189-L2HOr $(n=3)$. d Analysis of direct-repeat recombination frequencies in wild

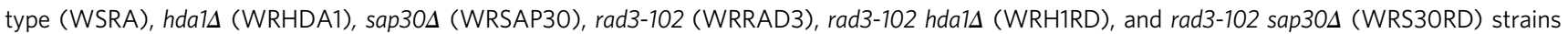
transformed with pSCH204 $(n=3)$. e Analysis of spontaneous recombination frequencies in wild type (WSRA), hda14 (WRHDA1), sap30د (WRSAP30), rad3-102 (WRRAD3), hda1s rad3-102 (WRH1RD), and sap304 rad3-102 (WRS30RD) strains transformed with pRS316-TINV ( $n \geq 3$ ). f Sensitivity to UV (40

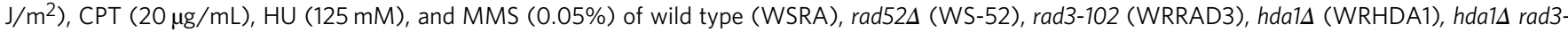

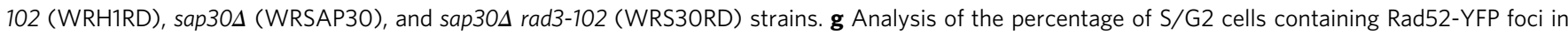

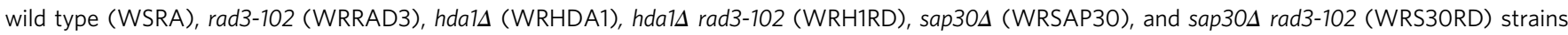
transformed with pWJ1344 in response to UV $\left(10 \mathrm{~J} / \mathrm{m}^{2}\right)$ or MMS $(0.01 \%)$. A representative image of a cell with a Rad52-YFP foci is shown $(n=3)$. h Analysis of pRS316 plasmid loss in wild type (WSRA), rad3-102 (WRRAD3), hda1s (WRHDA1), hda14 rad3-102 (WRH1RD), sap304 (WRSAP30), and sap304 rad3-102 (WRS30RD) strains $(n=3)$. i Analysis of the rate of gross chromosomal rearrangements (GCRs) at chromosome $V$ in wild type (YKJM1), hda1s (YKHDA1), and sap30د (YKSAP30) strains $(n=3)$. A diagram of the different systems is depicted in a-e, $\mathbf{h}$ and $\mathbf{i}$. Means and SEM are plotted in $\mathbf{a}-\mathbf{e}, \mathbf{g}$ and $\mathbf{h}$ whereas the mean is plotted in $\mathbf{i}^{\star \star} p \leq 0.05 ;{ }^{\star \star} p \leq 0.01 ;{ }^{\star \star \star} p \leq 0.001$ (two-tailed Student's $t$-test). Data underlying this figure are provided as Source Data file

plasmid-chromosome recombination after a replication-born $\mathrm{HO}$ break or a double-stranded DNA gap ${ }^{23}$, which is independent on the passage of the fork, implying that Hdal and Sap30 are only required to repair replication-born DSBs with the sister chromatid (Fig. 2b, c). We rather observed that sap30 $\Delta$, but not $h d a 1 \Delta$, increased this type of ectopic recombination events suggesting that failures to repair with the sister chromatid could lead to channeling of the repair to non-sister templates. Furthermore, both $h d a 1 \Delta$ and $\operatorname{sap} 30 \Delta$ led to a significant increase in spontaneous direct-repeat recombination ( $L$-lac $Z$ direct-repeat system) (Fig. 2d) in agreement with some of the spontaneous damage being channeled to ectopic DNA copies.

We also studied SCE levels when, instead of using the HO endonuclease, nicks were originated by the $\mathrm{Rad} 3$ endonuclease. For this, we took advantage of the rad3-102 allele, which impairs nucleotide excision repair (NER) postincision events leading to an accumulation of spontaneous ssDNA breaks ${ }^{31}$. rad3-102 induction of nicks was reflected in an increase in the levels of $\mathrm{Leu}^{+}$ recombinants with either the $L$-lacZ direct-repeat system (Fig. 2d) or the TINV-HO inverted-repeat system (Fig. 2e). These frequencies were significantly decreased in the absence of Hdal or Sap30 in the TINV-HO system, which measures SCE, but enhanced in the L-lacZ system further in agreement with these proteins having a defect in SCE and channeling the repair to ectopic DNA sequences. Moreover, hda1s rad3-102 and sap30 $\mathrm{rad3}-102$ were sensitive to UV, CPT, HU, and MMS, which enhance the possibilities of fork breakage (Fig. 2f), indicating that the defective repair of replicationinduced damage leads to cell death. These results suggest that the loss of Hda1 and Sap30 could enhance genetic instability. To address this possibility, we assayed genetic instability at a more general scale by studying plasmid loss, Rad52 foci accumulation, and GCRs. In all three cases, we observed an increase in $h d a 1 \Delta$ and sap30 $\Delta$ mutants with respect to wild-type cells (Fig. $2 \mathrm{~g}-\mathrm{i}$ ). In agreement with an increased occurrence of spontaneous unrepaired nicks, rad3-102 enhanced the number of cells with Rad52 foci and the frequency of plasmid loss (Fig. 2g, h). These effects were significantly higher in $h d a 1 \Delta$ and sap30 $\Delta$ mutants. Such additive effects of $h d a 1 \Delta$ and sap30 $\Delta$ mutations over rad3-102 on genetic instability were more evident after DNA damage, as observed in the frequency of cells with Rad52 foci after UV irradiation or MMS treatment (Fig. 2g). Altogether, our results support that $h d a 1 \Delta$ and sap30 $\Delta$ affect the repair of replication-born DSBs by the choice of a non-sister template leading to genetic instability.

Deacetylation by Hdal and Rpd3L is required for SCR. Hda1 is a class II HDAC that acts as the putative catalytic subunit of the
Hdal complex, and shares similarity with Rpd3 (ref. ${ }^{32}$ ). Therefore, it is possible that Hdal and Rpd3 work through similar or common pathways. To assay this possibility, we studied the effect of the absence of both Rpd3L and Hdal complexes. We repeated the SCE analysis with sap30 2 , hda1 $\Delta$, but now also including $\operatorname{rpd} 3 \Delta$ and the double mutant hda1 $\operatorname{rpd} 3 \Delta$. Genetic analysis of HO-induced recombination $(+\mathrm{HO})$ in the TINV-HO system revealed a similar decrease of around twofold in $r p d 3 \Delta$ and $h d a 1 \Delta$ $r p d 3 \Delta$ mutants whereas spontaneous $(-\mathrm{HO})$ recombination was increased to similar levels (Fig. 3a). Furthermore, no additive defect was observed in the hda1 $\Delta$ rpd3 $\Delta$ double mutant. We then performed a more complete time-point analysis of the repair reaction and changed our growing conditions from glycerollactate to raffinose to facilitate growth and DSB induction (Supplementary Fig. 2a). As shown in Fig. 3b, all mutants analyzed led to a similar decrease of around threefold in the efficiency of SCE respect to wild-type levels. These results suggest that Hdal and Rpd3L HDAC complexes might act through a common pathway for SCR. We therefore continued with rpd3 $\Delta$ to deepen into the possible mechanism behind the observed SCR defect.

To rule out any possible effects of Rpd3 in BIR, we used the previously reported system that measures the BIR intermediates between a full $\mathrm{HO}$-cut chromosome XV and an intact chromosome VI by $\mathrm{PCR}^{33}$. We observed the same levels of BIR in wild type and rpd3 $\Delta$ after 2,4 , and $6 \mathrm{~h}$ of $\mathrm{HO}$ induction revealing that $\mathrm{Rpd} 3$ is not involved in BIR (Fig. 3c). Therefore, Rpd3L is involved in SCR, and not in other replication-related recombination reactions such as BIR.

To test whether Rpd3 indeed acted through its HDAC function, we determined the effect of an Rpd3 deacetylase-dead mutant allele, $r p d 3-H 150 A^{34}$. Whereas the expression of wildtype Rpd3 (WT) complemented the SCE defect of rpd3 in both physical and genetic assays, the $r p d 3-H 150 \mathrm{~A}$ catalytically inactive allele was unable to rescue this phenotype and exhibited a strong SCE defect comparable to that of $r p d 3 \Delta$ cells transformed with an empty plasmid (Fig. 3d, e, Supplementary Fig. 2b). In agreement, spontaneous $(-\mathrm{HO})$ recombination levels were enhanced by rpd3-H150A (Fig. 3e). This result implies that the deacetylase function of $\operatorname{Rpd} 3$ is required for efficient SCR.

An FLP nickase-based system confirms the role for Rpd3 in SCR. HO endonuclease induces mainly nicks at the HOr site in any of the DNA strands and can also occasionally target both strands at the same time, although the frequency of these replication-independent DSBs is very low $(<2 \%)^{10}$. We therefore decided to create an improved version of the TINV-HO system in 
a

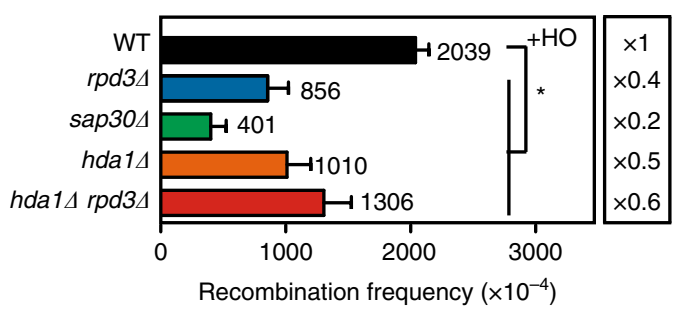

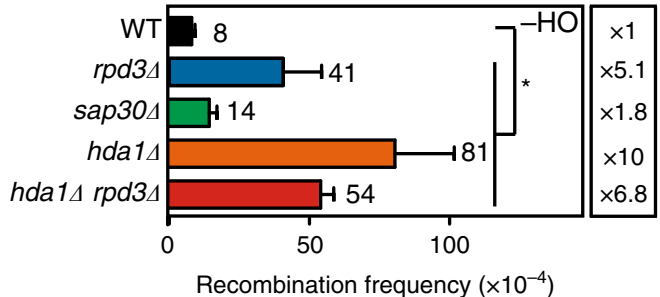

Recombination frequency $\left(\times 10^{-4}\right)$

b

WT

rpd34

sap304

hda14

hda14 rpd34

Gal (h): 01.534 .567 .5901 .534 .567 .59001 .534 .567 .59001 .534 .567 .5901 .534 .567 .59
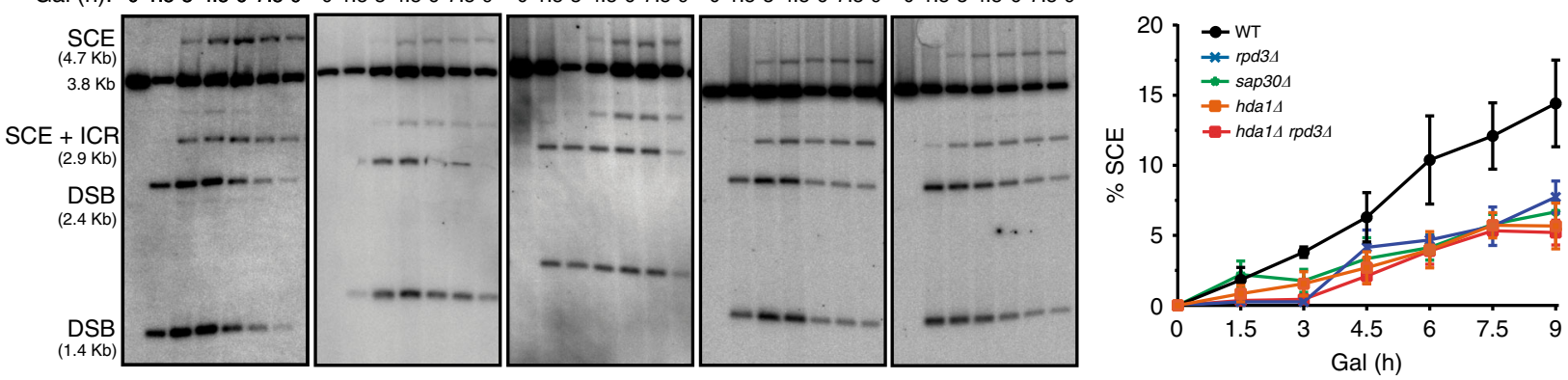

C

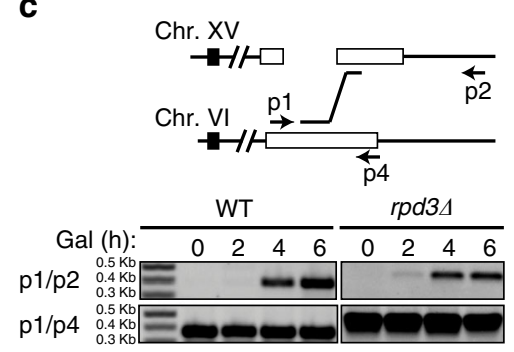

d

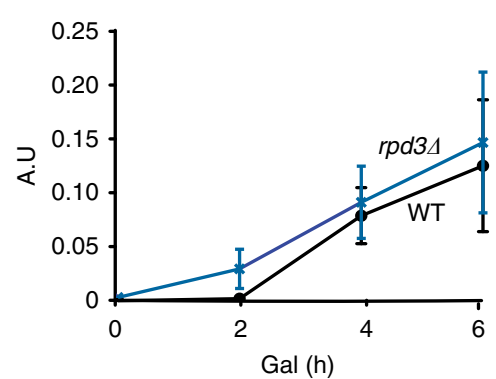

WT rpd34 rpd3-H150A

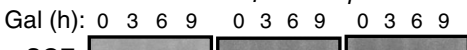

SCE

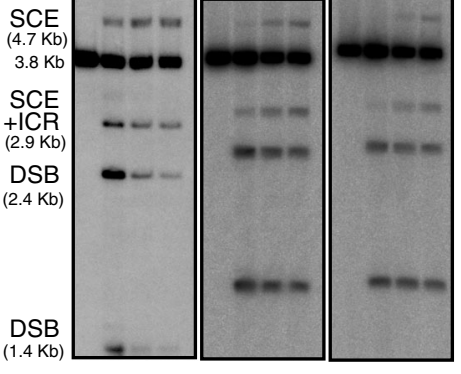

e
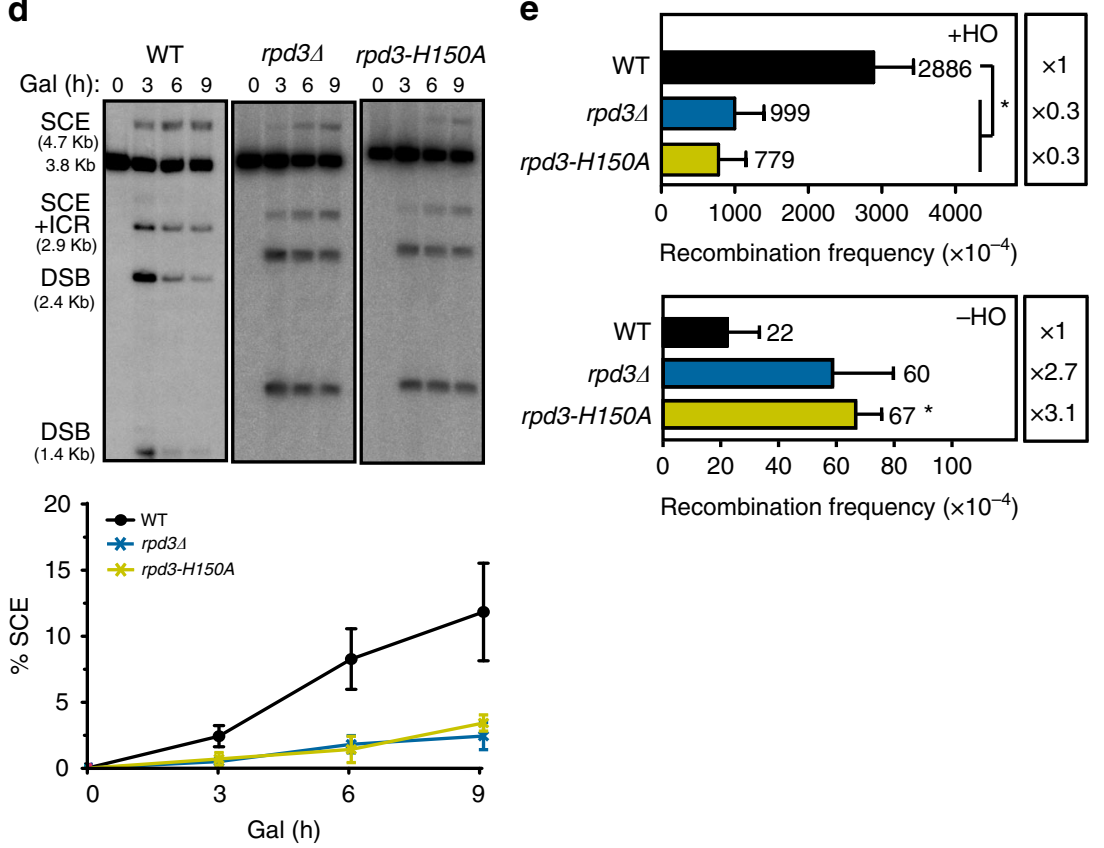

Recombination frequency $\left(\times 10^{-4}\right)$

Fig. 3 Deacetylation by Hda1 and Rpd3L impacts SCE through the same pathway. a Analysis of spontaneous ( $-\mathrm{HO})$ and $\mathrm{HO}$-induced (+HO)

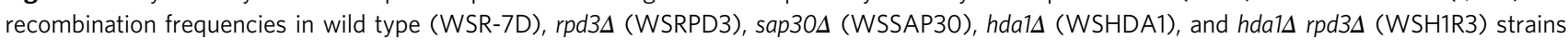
transformed with pRS316-TINV $(n \geq 3)$. b Representative Southern blots and quantification of the 4.7-kb SCE fragments during time-course experiments

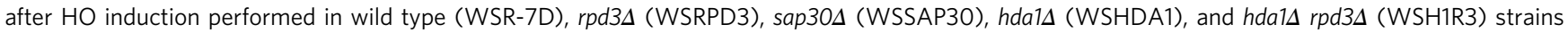
transformed with pRS316-TINV ( $n \geq 3$ ). c Quantification of BIR intermediates in wild type (JFR-4) and rpd3A (JFRPD3) strains. A scheme of the BIR reaction is shown with the primers depicted as arrows. PCR with p1 and p2 oligos were used for the detection of BIR intermediates produced when the HO-cut chromosome III invades the homologous ACT1 intron sequence on chromosome VI. PCR with p1 and p4 primers was used as a control. A representative image of the PCR products is shown $(n=4)$. $\mathbf{d}$ Representative Southern blots and quantification of the 4.7-kb SCE fragments during a time-course

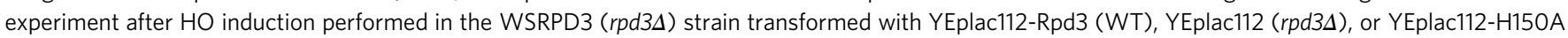
(rpd3-H150A) plasmids $(n \geq 3)$. e Analysis of spontaneous $(-\mathrm{HO})$ and HO-induced $(+\mathrm{HO})$ recombination frequencies in the WSRPD3 (rpd34) strain transformed with either YEplac112-Rpd3 (WT), YEplac112 (rpd34), or YEplac112-H150A (rpd3-H150A) and the pRS316-TINV plasmids ( $n \geq 4)$. Means and SEM are plotted in all panels. ${ }^{*} p \leq 0.05$ (two-tailed Student's $t$-test). In a and $\mathbf{d}$, the $4.7-\mathrm{kb}$ band is specific for SCE whereas the 2.9-kb band can result from SCE and other inter-chromatid recombination (ICR) events. The 3.8-kb band corresponds to the intact plasmid and 1.4 and 2.4-kb bands to the DSBs. See also Supplementary Fig. 2. Data underlying this figure are provided as Source Data file 
a

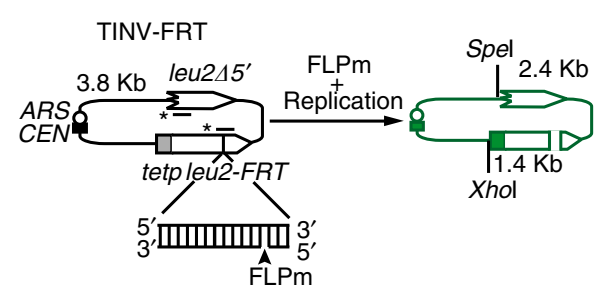

Native

(DSB)

Alkaline

(total breaks)
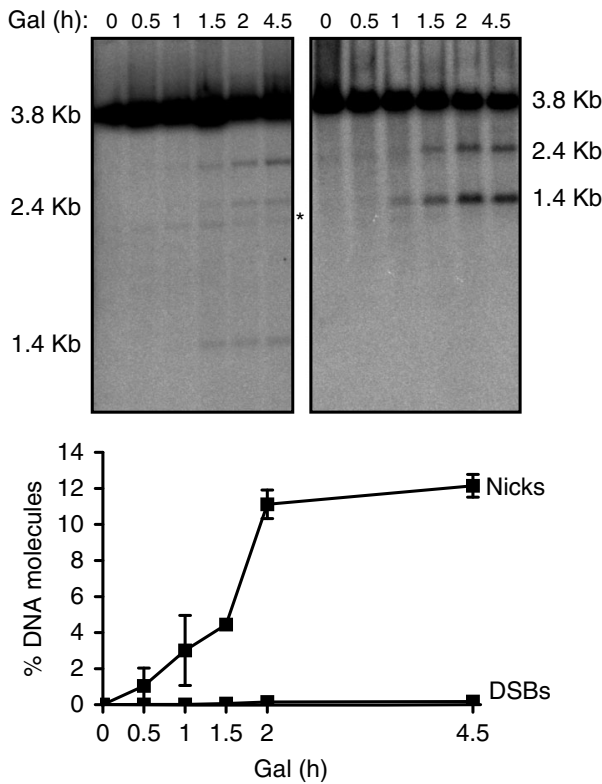

C

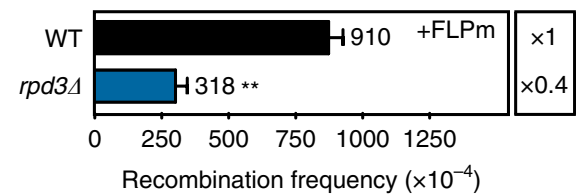

b
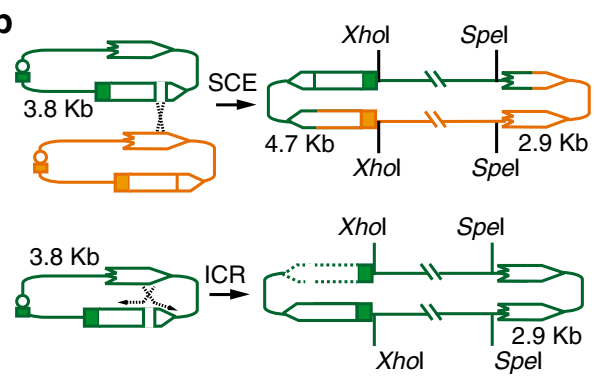

WT

rpd34
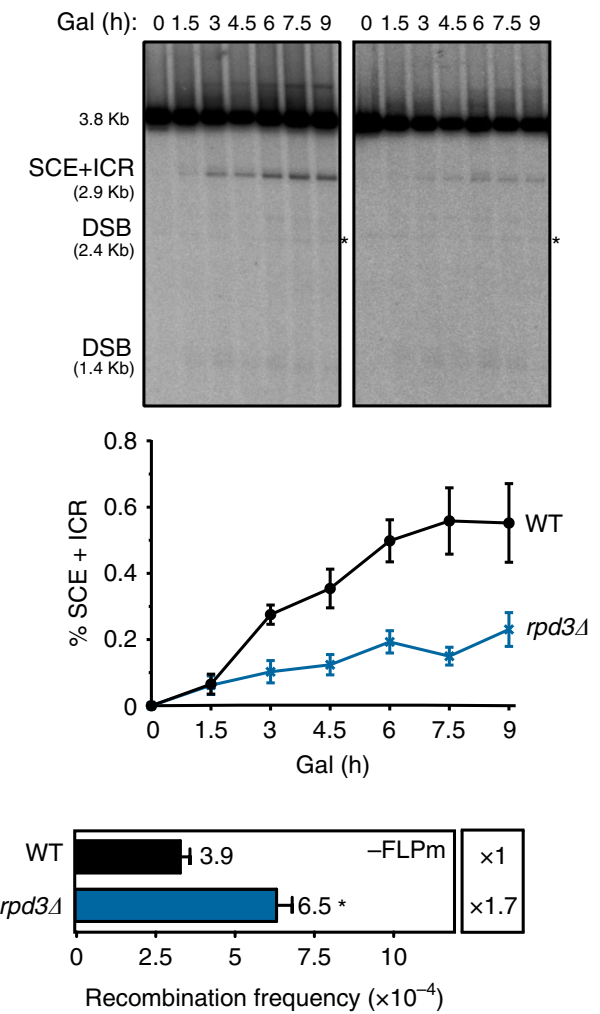

Fig. 4 An FLP nickase-based system confirms the role for Rpd3 in SCR. a Analysis of the formation of nicks and DSBs after FLPm induction in the wild-type (WFLP) strain transformed with the PTINV-FRT plasmid. A scheme of the TINV-FRT system before and after the FLPm-induced nick is converted to a DSB by replication and representative Southern blots of the time-course analysis after FLPm induction after native and alkaline gel electrophoresis are shown $(n=2)$. b Representative Southern blots and quantification of the 2.9-kb SCE + ICR fragments during a time-course experiment after FLPm induction performed at $37^{\circ} \mathrm{C}$ in wild type (WFLP) and $r p d 3 \Delta$ (WFRPD3) strains transformed with pTINV-FRT ( $\left.n \geq 3\right)$. A scheme of the TINV-FRT system and SCE and ICR intermediates produced after FLPm-induced replication-born DSBs is shown. c Analysis of spontaneous ( $-F L P m)$ and FLPm-induced (+FLPm) recombination frequencies at $33^{\circ} \mathrm{C}$ in wild type (WFLP) and rpd3A (WFRPD3) strains transformed with pTINV-FRT $(n=3)$. In $\mathbf{a}$ and $\mathbf{b}$, fragments generated after Xhol-Spel digestion are indicated with their corresponding sizes in kb and were detected by Southern blot hybridization with a LEU2 probe (line with an asterisk). Asterisks beside Southern blots indicate non-specific hybridization. Means and SEM are plotted in all panels. In $\mathbf{c},{ }^{\star} p \leq 0.05 ;{ }^{\star \star} p \leq$ 0.01 (two-tailed Student's $t$-test). See also Supplementary Fig. 3A. Data underlying these figures are provided as Source Data file

which we introduced an FRT site instead of the HOr site (TINVFRT system). FRT is the target for the flipase recombinase (FLP), a mutated version of which (FLP-H305L, FLPm) causes an irreversibly protein-bound nick ${ }^{35}$ and was previously used to study $\mathrm{BIR}^{36}$. Two hours after FLPm induction, $11 \%$ of the molecules were nicked, whereas less than $0.2 \%$ of molecules were cut in both strands, as we determined by the use of native and alkaline gel electrophoresis in which the 2.4 and 1.4-kb bands corresponded to either only DSBs in the native or to total breaks in the alkaline gel (Fig. 4a). These DSB levels were almost 20-fold lower than those obtained with the TINV-HO system, consistent with a notable improvement in the enrichment of replication-born DSBs versus other type of breaks. As a consequence of this decrease in the total percentage of DSBs, it was necessary to increase fourfold the amount of DNA loaded on the gels and the strong signal of the $3.8-\mathrm{kb}$ band corresponding to the fully linearized plasmid hindered the 4.7-kb SCE-specific band, impeding its reliable quantification. We therefore quantified the $2.9-\mathrm{kb}$ band (SCE + ICR), mostly arising as a consequence of SCE intermediates but that can also reflect some intrachromatid recombination (ICR) events such as BIR intermediates ${ }^{6}$. As shown in Fig. $4 \mathrm{~b}$, the levels of SCE + ICR strongly decreased in the absence of Rpd3. As a consequence of repair, DSBs levels decreased after $3 \mathrm{~h}$ of FLPm induction in the wild type but they dropped more slowly in $r p d 3 \Delta$ cells (Supplementary Fig. 3a). When FLPm was induced, the genetic detection of $\mathrm{Leu}^{+}$recombinants increased by two orders of magnitude (Fig. 4c, +FLPm versus -FLPm) further confirming the formation of DSBs. FLPm-induced recombination levels 
$(+\mathrm{FLPm})$ dropped in rpd3 3 cells, but increased under spontaneous conditions (-FLPm). Altogether, these results validate the TINV-FRT as an improved system to measure the specific repair of replication-born DSBs through SCR and further confirm a role for $\mathrm{Rpd} 3$ in this process.

Similar efficiency of SCR at the leading and lagging strands. In addition to improving the specificity for nicks, the TINV-FRT system allows to direct the ssDNA break to either the Watson or the Crick strand, depending on the orientation of the FRT site. We therefore created two versions of the system to study the repair of nicks made in either the leading or the lagging strands by inserting FRT in each of the two opposite orientations (FRTd and FRTg, respectively) (Fig. 5a). Analysis of the DSBs' appearance and the efficiency of repair by using these two TINV-FRT systems revealed similar results for all time-points in wild-type cells (Supplementary Figs. $3 \mathrm{~b}$ and $5 \mathrm{a}$ ). This indicates that there are no major differences in the kinetics of SCE repair of DSBs arising as a consequence of a nick on either the leading or lagging strand.

In the genetic analysis, although the spontaneous recombination frequencies (-FLPm) were similar in both constructs $\left(17 \times 10^{-5}\right.$ and $\left.14 \times 10^{-5}\right)$, the induction of the FLPm nickase $(+\mathrm{FLPm})$ led to a twofold higher increase in the TINV-FRTd leading strand system (Fig. 5b). Since we detected no difference in the kinetics of SCE repair between both constructs (Fig. 5a), this difference must account for non-SCE recombination events. Importantly, $r p d 3 \Delta$ mutant led to a defective repair in both leading and lagging strands constructs as determined by both physical and genetic analyses (Fig. 5a, b), whereas spontaneous recombination levels were significantly higher (Fig. 5b), in agreement with our previous observations. Therefore, we conclude that DSBs arising as a consequence of forks that encounter a nick in either the leading or lagging strand are repaired by SCE with the same efficiency and require $\mathrm{Rpd} 3$.

Rpd3 and Hdal promote cohesin loading and chromatid cohesion. We investigated the possible mechanisms by which Rpd3L could be required for efficient SCR. For this, we first performed a genetic analysis of the spontaneous and HO-induced recombination levels in double mutant strains carrying $r p d 3 \Delta$ in combination with mutations in genes encoding other factors previously reported to be involved in SCR (Rad51, Sae2, Sgs1, Mus81, Rrm3, Hst3, and Scc1). Double mutant combinations in $R P D 3$ and general HR factor genes (RAD51, SAE2, SGS1, MUS81) resulted in a further decrease in $\mathrm{HO}$-induced recombination levels but no major changes in spontaneous recombination with respect to the single mutants (Fig. 6a, Supplementary Fig. 4a). This synergistic behavior points to a role for Rpd3 in SCR through a mechanism not related to general HR and is consistent with the spontaneous hyper-recombination phenotype observed (Fig. 2). By contrast, the levels of $\mathrm{HO}$-induced recombination were significantly higher in $r p d 3 \Delta \mathrm{rrm} 3 \Delta$ and $r p d 3 \Delta$ hst $3 \Delta$ than in either single mutant (Fig. 6a, upper panel), and the recombination frequency was already spontaneously augmented in $r p d 3 \Delta \mathrm{rrm} 3 \Delta$ (Supplementary Fig. 4a), likely reflecting higher levels of unrepaired damage. Instead, the combination of $r p d 3 \Delta$ with the $s c c 1$ 73 thermosensitive mutation, which impairs cohesion ${ }^{37}$ and $\mathrm{SCE}^{10}$, showed no significant changes in HO-induced or spontaneous recombination levels when assayed at the semipermissive temperature $\left(33^{\circ} \mathrm{C}\right)$ (Fig. 6a, lower panel). This suggests a possible epistatic relationship between these two factors. Genetic and physical analysis of FLPm-induced or spontaneous recombination showed analogous results, with the double rpd3 $\Delta$ scc1-73 mutant presenting similar levels of FLPm-induced recombination frequency and SCE intermediates than either single mutant (Fig. 6b, c, Supplementary Fig. 4b, c). These results suggested a cohesin-related function as the responsible for the SCR defect observed in $r p d 3 \Delta$.

Therefore, we analyzed cohesin levels in wild type and $r p d 3 \Delta$ cells by chromatin immunoprecipitation (ChIP) experiments of the MYC-tagged cohesin subunit Scc1 (Scc1-MYC). Given that cohesins are also recruited upon DSBs, we analyzed Scc1-MYC occupancy before and after $\mathrm{HO}$ induction $(-\mathrm{HO}$ and $+\mathrm{HO}$, respectively) in the same sequences and conditions used to study SCR (Fig. 7a). Although cohesin loading was not significantly enhanced by HO induction, likely due to the low efficiency of HO cleaving the HOr site, we detected a threefold defect in cohesin loading before and after $\mathrm{HO}$ induction in the absence of Rpd3 (Fig. 7a) suggesting that $r p d 3 \Delta$ compromises cohesin loading regardless of DSB induction. We also monitored four regions of chromosome III, including ARS305a, ARS305b, SYP1, and the centromere (CEN3), prone to cohesin enrichment ${ }^{38}$. As shown in Fig. $7 \mathrm{~b}, r p d 3 \Delta$ caused a two- to threefold significant decrease of Scc1-MYC immunoprecipitation in all four regions analyzed in spontaneous conditions. Hence, we confirmed that Rpd3 loss causes a global defect in cohesin loading in undamaged conditions. Similar results were obtained after $\mathrm{HO}$ induction (Supplementary Fig. 5a).

We hypothesized that the lower loading of cohesins to chromatin should impact negatively on sister chromatid cohesion. Therefore, we monitored cohesion directly through a previously described system which is based on a LacI::GFP fusion and a multi-copy LacO-binding site placed at the CEN3 (ref. ${ }^{39}$ ). In agreement with the presence of one single chromatid in G1, few cells presented two GFP foci in a-factor synchronized cells (Supplementary Fig. 5b, c). This percentage increased to 8 in G2/M in wild-type cells but to almost $23 \%$ in rpd3 4 cells indicating a clear defect in sister chromatid cohesion in the absence of Rpd3 (Fig. 7c). Importantly, and consistent with the epistatic relationship between $h d a 1 \Delta$ and rpd3 $\Delta$ regarding SCR, hda1 $\Delta$ showed a similar defect in sister chromatid cohesion (Fig. 7c). However, when we tested other SCR-defective mutants such as hst $3 \Delta$ and $\mathrm{rrm} 3 \Delta$, for which our double mutant analysis (Fig. 6) suggested that they act via a different mechanism, we only observed a slight but not significant decrease in either Sccl occupancy (Fig. 7b) and sister chromatid cohesion (Fig. 7c). Further in agreement with the epistatic effects observed in recombination, the combination of Rpd3 loss with deletions in general HR factors (sgs1 $\Delta$ ) or specific replication/SCR factors $(\mathrm{rrm} 3 \Delta)$ did not enhance this cohesion defect (Fig. 7c). Moreover, the loss of Rpd3 was epistatic with $\operatorname{scc} 1-73$, as expected from both factors affecting cohesion through a common pathway (Fig. 7c). To further determine whether the HDAC function of Rpd3 was behind its cohesion defect, we performed this assay with the rpd3-H150/ $1 A$ deacetylase-dead mutant allele ${ }^{40}$. As observed for SCE (Fig. 3d, e), whereas the expression of wild-type Rpd3 (WT) complemented the cohesion defect of $r p d 3 \Delta$, the catalytically inactive allele showed a cohesion defect similar to that of $r p d 3 \Delta$ cells transformed with an empty plasmid (Fig. 7d), implying that Rpd3 affects sister chromatid cohesion through its HDAC activity.

Finally, it is worth noting that $\mathrm{Hda} 1$ and $\mathrm{Rpd} 3$ have sequence similarity to Hos1 (ref. ${ }^{32}$ ), a deacetylase involved in Smc3 deacetylation after the S-phase to promote the separation of the sister chromatids ${ }^{41,42}$. Although Hdal and Rpd3 have been reported not to be able to deacetylate Smc3 in vitro ${ }^{41}$, our results could be explained if SCR required cohesin deacetylation and Hda1 and/or Rpd3 could act directly on cohesins 
a

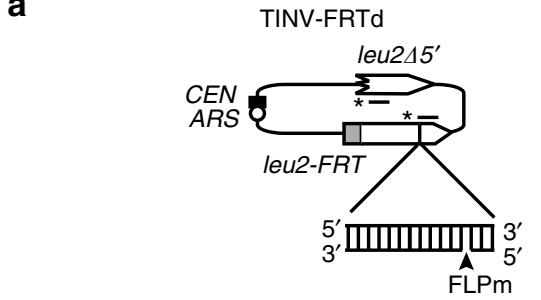

TINV-FRTg

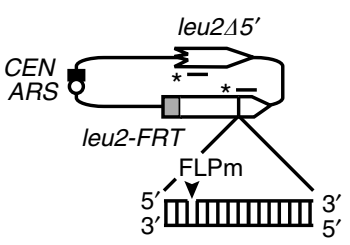

WT
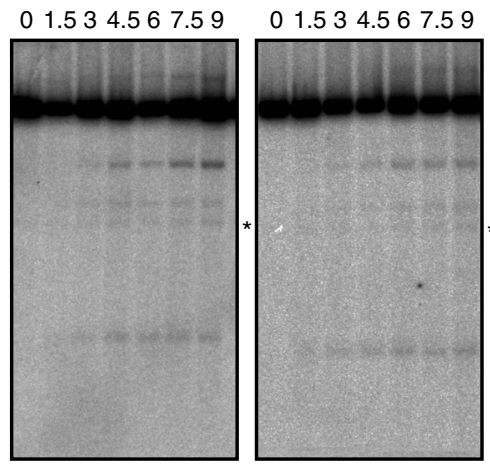

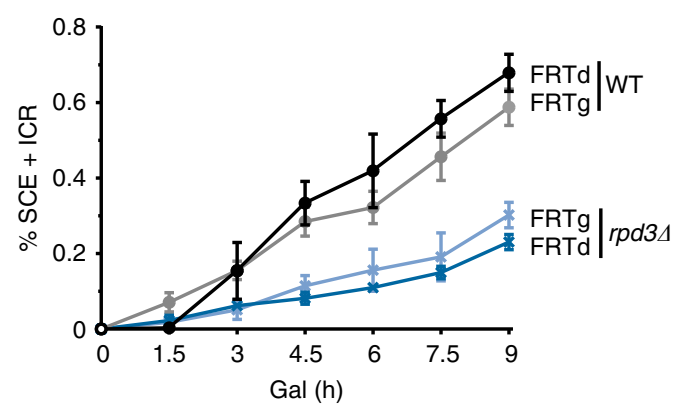

b
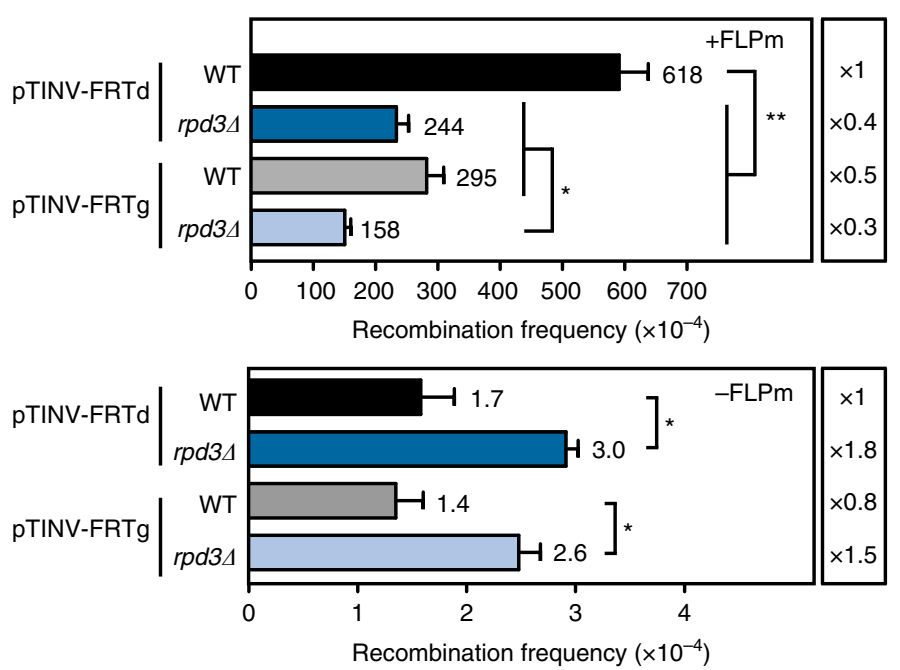

Fig. 5 Similar efficiency of SCR at the leading and lagging strands. a Representative Southern blots and quantification of the 2.9-kb SCE + ICR fragments during a time-course experiment after FLPm induction in wild type (WFLP) and rpd3A (WFRPD3) strains transformed with pTINV-FRTd or pTINV-FRTg $(n=3)$. Schemes of the TINV-FRTd and TINV-FRTg systems are shown. Other details as in Fig. 4b. $\mathbf{b}$ Analysis of spontaneous ( - FLPm) and FLPm-induced $(+F L P m)$ recombination frequencies wild type (WFLP) and rpd3A (WFRPD3) strains transformed with pTINV-FRTd or pTINV-FRTg $(n=3)$. Means and SEM are plotted in all panels. In $\mathbf{b},{ }^{\star} p \leq 0.05 ;{ }^{\star \star} p \leq 0.01$ (two-tailed Student's $t$-test). See also Supplementary Fig. 3b. Data underlying this figure are provided as Source Data file

in vivo. If that were the case, we would expect that hos $1 \Delta$ cells should have an SCE defect. As shown in Supplementary Fig. 5d, however, the efficiency of SCE was not affected in hos $1 \Delta$ as it was in $r p d 3 \Delta$, even though DSBs were induced with lower efficiency in hos $1 \Delta$. This result indicates that cohesin deacetylation by itself is not required for efficient SCE, and support a role for the deacetylation of histones, rather than cohesins, in the repair of replication-born DSBs.

Altogether, our results demonstrate that Rpd3L and Hda1 are required for the efficient repair of replication-born DSBs through 

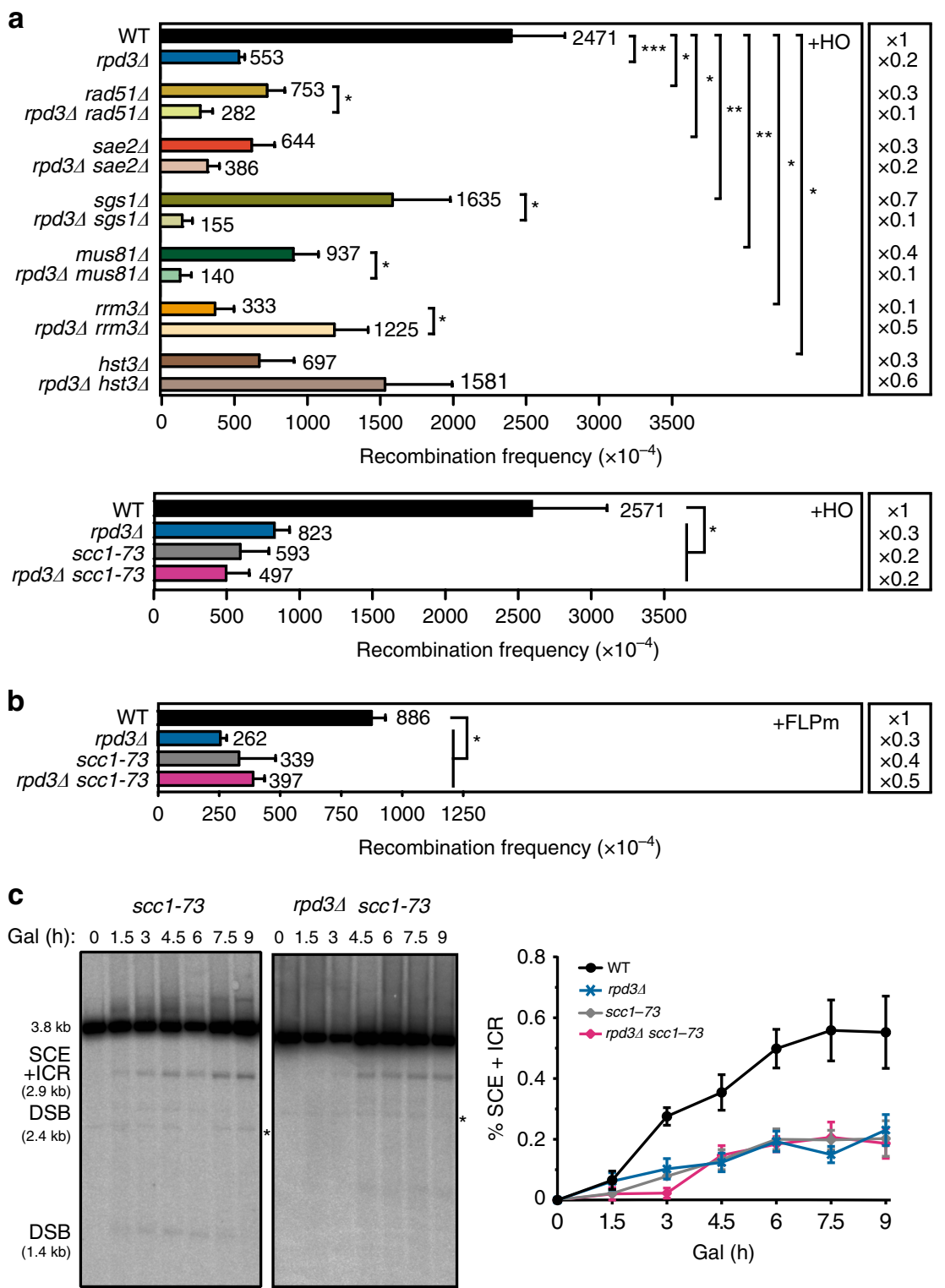

Fig. $6 \mathrm{SCE}$ levels in rpd3 $\Delta$ in combination with other SCR-affected mutants. a Analysis of $\mathrm{HO}$-induced recombination frequencies at $30^{\circ} \mathrm{C}$ (upper panel)

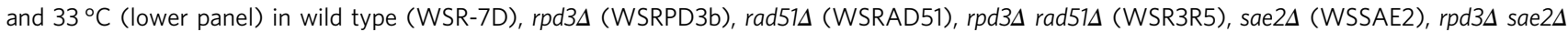

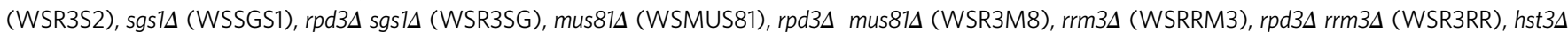

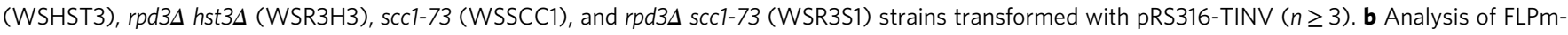
induced recombination frequencies at $33^{\circ} \mathrm{C}$ in wild type (WFLP), rpd3 (WFRPD3), scc1-73 (WFSCC1), and rpd34 scc1-73 (WFR3S1) strains transformed with pTINV-FRT $(n=3)$. c Representative Southern blots and quantification of the 2.9-kb SCE + ICR fragments during a time-course experiment after FLPm induction performed at $37^{\circ} \mathrm{C}$ in wild type (WFLP), rpd3 (WFRPD3), scc1-73 (WFSCC1), and rpd3A scc1-73 (WFR3S1) strains transformed with pTINV-FRT $(n \geq 3)$. Other details as in Fig. 4b. Means and SEM are plotted in all panels. In $\mathbf{a}$ and $\mathbf{b}{ }^{\star} p \leq 0.05$; ${ }^{\star \star} p \leq 0.01$ (two-tailed Student's $t$-test). See also Supplementary Fig. 4. Data underlying this figure are provided as Source Data file

SCR via a role for histone deacetylation in sister chromatid cohesion (Fig. 7e).

\section{Discussion}

We show here an epigenetic regulation of the repair of replicationborn DSBs by the modulation of sister chromatid cohesion. Through the use of a previously reported TINV-HO assay and an FRT/FLPm-based system for the study of repair of replication-born DSBs, we define a specific role of Rpd3L and HDACs in SCR. We demonstrate that this occurs through a role of these HDACs in sister chromatid cohesion by facilitating the general loading of cohesins (Fig. 7). Since cohesion between sister chromatids influences the efficiency of SCR, the major repair pathway for DSBs arising at replication forks, the epigenetic regulation of cohesion has a strong impact on the repair of broken forks and the maintenance of genome integrity (Figs. 1-3).

The TINV-FRT system developed in this study strongly improved the efficiency of nicks versus unspeficic DSBs (Fig. 4a) and enabled to study repair of leading versus lagging strand forks (Fig. 5). We showed that nicks in the leading or lagging strand encountered by a fork converted into DSBs with a similar kinetics (Supplementary Fig. 3b) and were repaired with the same efficiency (Fig. 5). These results not only validate all our previous results obtained with the TINV-HO system in which nicks could occur randomly on either strand, but ratify TINV-FRT systems for the physical analysis of repair of replication-born DSBs by SCR. 
a

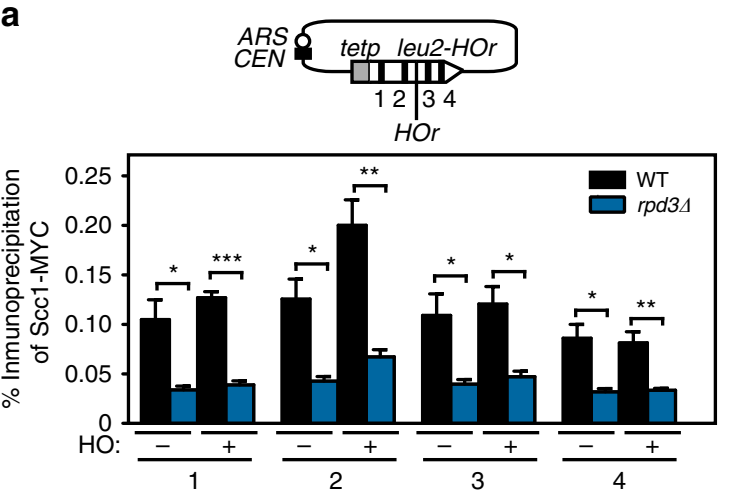

C

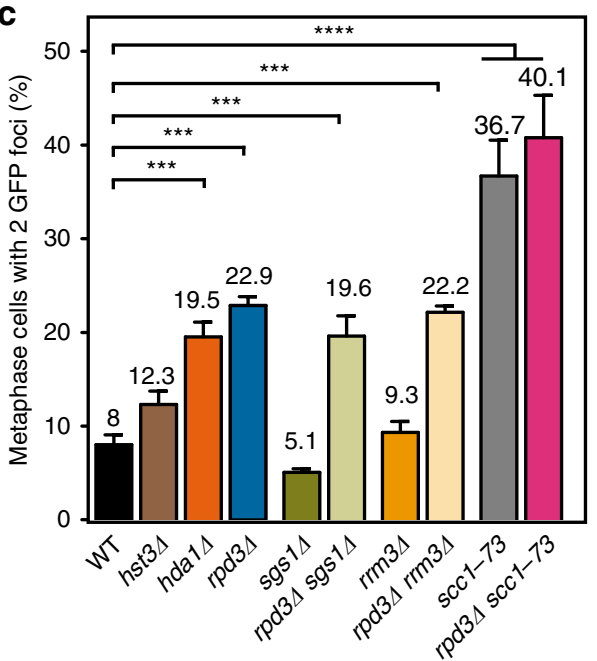

e WT

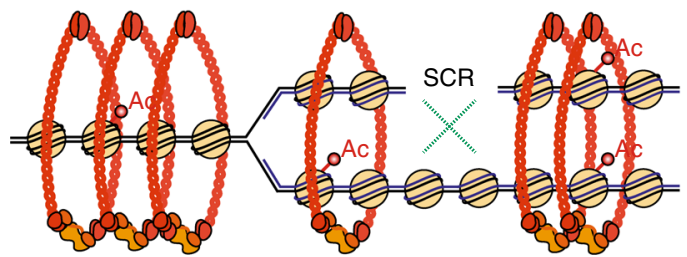

b
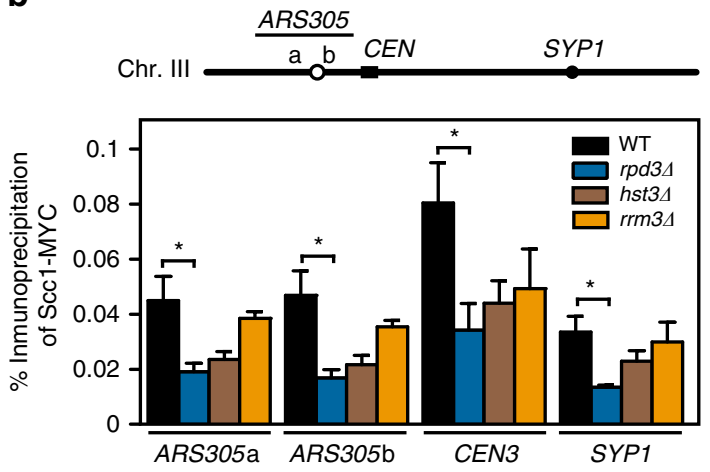

d

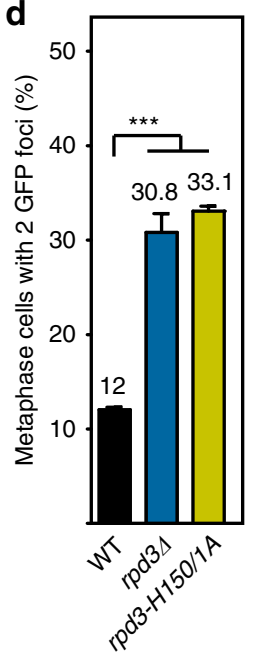

Rpd3L or Hda1 loss

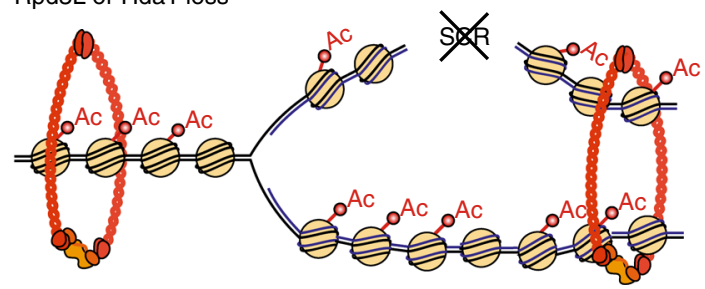

Fig. 7 Rpd3 and Hda1 promote cohesin loading and sister chromatid cohesion. a ChIP analysis of Scc1-MYC occupancy in wild type (WSM) and rpd3A (WSMR3) strains after glucose $(-\mathrm{HO})$ or galactose $(+\mathrm{HO})$ addition for repression or activation of the $\mathrm{HO}$ endonuclease. A scheme of the different regions analyzed along the leu2 gene of pCM189-L2HOr is depicted on top ( $n \geq 3$ ). b ChIP analysis of Scc1-MYC occupancy in wild type (WSM), rpd34 (WSMR3),

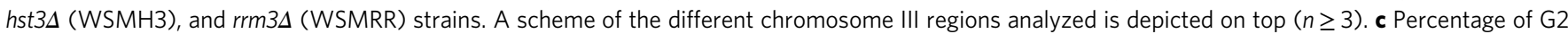
(nocodazol) arrested cells that have lost sister chromatid cohesion at centromere III, as indicated by the appearance of 2 GFP foci, in wild type (SBY885),

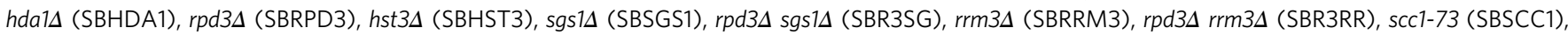
and $r p d 3 \Delta$ scc1-73 (SBR3S1) ( $n \geq 3$ ). d Percentage of G2 (nocodazol) arrested cells that have lost sister chromatid cohesion at centromere III, as indicated by the appearance of 2 GFP foci, in SBRPD3 (rpd34) strain transformed with PEN149 (WT), pRS315 (rpd34), or PEN153 (rpd3-H150/1A) plasmids ( $n=3$ ). e A model to explain the role of Rpd3 in the repair of replication-born DSBs by SCR. In wild-type cells (WT), deacetylated chromatin supports cohesin loading, and thus sister chromatid cohesion, to favor SCR. By contrast, in the absence of Rpd3L or Hda1-mediated histone deacetylasaes, hyperacetylated chromatin would impair sister chromatid cohesion and hence affect the choice of the sister as a template for DSB repair. Means and SEM are plotted in a-d. In $\mathbf{a}-\mathbf{d},{ }^{\star} p \leq 0.05 ;{ }^{\star \star} p \leq 0.01 ;{ }^{\star \star \star} p \leq 0.001$ (two-tailed Student's $t$-test). See also Supplementary Fig. 5. Data underlying this figure are provided as Source Data file

Chromatin is a major determinant for all biological processes occurring on DNA. Here we define Rpd3L and Hda1-mediated histone deacetylation as a layer of regulation specific for SCR. Interestingly, Rpd3 and other HDACs are recruited to DSBs during HR repair already suggesting that they could have some roles in regulating $\mathrm{HR}^{43}$. We show that deacetylation is required for the choice of the sister chromatid to repair replication-born DNA breaks on either strand (Fig. 5). Indeed, the same SCR defect was observed in a deacetylase-dead mutant of Rpd3
(Fig. 3d, e). Therefore, histone deacetylation emerges as a major regulator of DSB repair in eukaryotes via SCR. Supporting the relevance of histone deacetylation and SCR in preventing genome instability, sap $30 \Delta$ and hda $1 \Delta$ mutants led to increased levels of unrepaired Rad52 foci, GCRs, and plasmid loss, and this was further enhanced when replication-born breaks were promoted by the rad3-102 mutation (Fig. 2).

Importantly, the mechanism by which the loss of HDAC impairs SCR is linked to a general effect caused on cohesin 
loading, which we observed both at the break site and in all other genomic regions analyzed (Fig. 7). Although an influence of de novo loading of cohesins to DSBs in SCR was previously reported $^{15,19-21}$, the fact that Rpd3L loss affected cohesin occupancy both at the break site as well as at the centromere and chromosome arms and in the absence of DSB induction (Fig. 7a, b) provides evidence for a role of general cohesin loading in SCR regardless of breakage. The defective cohesin loading has a direct impact on cohesion, as we demonstrate at the centromere in the absence of Rpd3 or Hdal and in the catalytically inactive mutant of RPD3 (Fig. 7c, d). This is unlikely related to a direct role on acetylated cohesins, as SCR is not impaired in the absence of the Hos1 cohesin deacetylase (Supplementary Fig. 5d). Hence, we support a model in which deacetylated chromatin stimulates cohesin loading, and thus sister chromatid cohesion, to favor SCR (Fig. 7e). By contrast, hyperacetylated chromatin would impair sister chromatid cohesion and affect the choice of the sister as a template to repair broken forks and likely any DSB occurring during S/G2. Chromatin appears, therefore, as a critical parameter for the stimulation of sister chromatid cohesion by controlling the levels of cohesins sitting on chromosomes.

In agreement with chromatin influencing cohesion, chromatin remodelers have been reported to influence cohesion ${ }^{44-46}$. Nonetheless, and although chromatin modification was hypothesized to impact cohesion ${ }^{47}$, the only hint of a conection between chromatin modifications and sister chromatid cohesion comes from the observation that heterochromatin promotes cohesin association in fission yeast and higher eukaryotes ${ }^{48-50}$. Similarly, cohesion is enhanced in budding yeast heterochromatin-like silent chromatin, which is mediated by Sir2 class III HDAC, although this is independent on its HDAC function ${ }^{51,52}$.

The connection between histone deacetylation and general cohesion uncovered here opens the possibility that other epigenetic marks could act at different levels in the regulation of cohesion and thus specifically impact SCR but not other mechanisms of DNA repair. Nonetheless, this does not exclude that other pathways beside sister chromatid cohesion could also impact the repair of broken forks by SCR. Indeed, a significant defect was not detected in hst3 $\Delta$ or $r r m 3 \Delta$ mutants (Fig. 7), previously reported to specifically affect SCR, indicating that these two factors impact SCR likely through a different pathway. In this sense, it is worth mentioning that although a centromeric

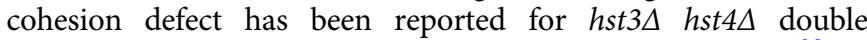
mutants, this was not observed in hst $3 \Delta$ single mutants ${ }^{39}$ in agreement with our results. Identifying how different factors control SCR besides sister chromatid cohesion should help us understand the mechanisms by which SCR is preferentially used over other types of homology-dependent DSB repair ptahways.

Hda1 and Rpd3L-deacetylated chromatin appears therefore as the milieu needed for the appropriate cohesion in the S-phase to promote SCR and ensure the accurate repair of broken forks. Interestingly, cohesins accumulate at stalled replication forks and seem required for efficient fork progression after DNA damage ${ }^{53,54}$. However, it is worthy to note that the action of Rpd3L was reported to be toxic in the S-phase checkpoint mutants ${ }^{55}$. Our results imply that although histone deacetylation can have deleterious consequences at stalled forks, it can also be beneficial for repair and fork progression and suggest that histone acetylation levels must be finely regulated to accomplish proper genome duplication.

Likely as a consequence of the innacurate repair of broken forks, we have observed several indicators of increased genetic instability in the absence of Hdal and Rpd3L, such as ectopic recombination, plasmid loss, and GCRs (Fig. 2). Suggesting a possible conservation of this connection between histone acetylation and SCR in human cells, depletion of the human homologs of Sap30 and Sin3 also lead to increased DNA damage ${ }^{56}$.
Interestingly, this damage was related to increased replication fork blockage caused by DNA-RNA hybrids ${ }^{56}$. It is thus possible that the Sin3A deacetylase complex also regulates repair of replication-born DSBs, whether or not induced by RNA-DNA hybrids, by promoting SCR through cohesion in human cells. Noteworthy, histone acetylation levels can impact the choice between HR and NHEJ pathways by influencing the chromatin association of the key repair factors BRCA1 and 53BP1 (ref. ${ }^{57}$ ) providing an example of the influence of chromatin structure in DSB repair pathway choice. Supporting that sister chromatid cohesion is essential for DNA repair also in higher eukaryotes, Scc1 depletion causes DNA damage sensitivity in DT40 and human cells ${ }^{58-60}$. Interestingly, ablation of cohesion in human cells was reported to cause GCRs as a consequence of the ligation of distal DNA breaks ${ }^{61}$ and impaired cohesion was related to chromosomal instability and cancer ${ }^{62}$. These observations suggest that the regulation of SCR by cohesion might be conserved in higher eukaryotes and agree with our interpretation that the cohesion defects observed in the absence of Hda1 and Rpd3L are behind their genetic instability phenotypes.

Our study, therefore, not only uncovers a specific role of histone deacetylation in the repair of replication-born DSBs with the sister chromatid, but indicates a key role of histone acetylation levels in the loading of cohesins. The involvement of Hdal and Rpd3 in cohesion might contribute to understand why patients with chromatin-related mutations show overlapping phenotypes with human diseases caused by mutations in cohesin-related genes (cohesinopaties), such as Cornelia de Lange syndrome ${ }^{63}$. Similarly, it is possible that some of the features of cohesinopaties are caused by defects in SCR or by the subsequent genetic instability. Strikingly, although cohesinopaties have not been related to cancer predisposition ${ }^{64,65}$, cells from Cornelia de Lange patients show increased DNA damage sensitivity, particularly when cells are exposed to damage after replication ${ }^{66}$. Our study opens, thus, perspectives to understand the role of epigenetic modifications in the preservation of genome integrity and cancer prevention and ascertain the role of sister chromatid cohesion in promoting SCR as the most prominent DSB repair pathway.

\section{Methods}

Yeast strains and media. Yeast strains used in this study are listed and described in Supplementary Table 1.

The WFLP strain was generated by MET17 gene replacement with the GALFLPH250L::HPHMX6 fragment from pGAL-FLPH250L. pGALFLPH250L was previously built by inserting the PvuII GAL-FLPH250L fragment from pBISGalkFLP67 into PvuII-digested pFA6aHPHMX6 (ref. ${ }^{68}$ ).

Media used in this study: YPAD (1\% yeast extract, $2 \%$ bacto-peptone, $2 \%$ glucose, $20 \mathrm{mg} / \mathrm{L}$ adenine), SD ( $0.17 \%$ yeast nitrogen base (YNB) without amino acids nor ammoninum sulfate, $0.5 \%$ amonium sulfate and supplemented with amino acids. The absence of amino acid/s is specified when required), SC (SD containing $2 \%$ glucose). SGal (SD containing $2 \%$ filtered-galactose), SRaf (SD containing $2 \%$ raffinose), SGL (SD containing $3 \%$ filtered-glicerol and $2 \%$ sodium lactate), SPO ( $1 \%$ potassium acid, $0.1 \%$ yeast extract, $0.005 \%$ glucose), and FOA (S with half concentration of uracil $(10 \mathrm{mg} / \mathrm{L}), 0.1 \mathrm{~L}$-proline instead of amonion sulfate and $50 \mathrm{mg} / \mathrm{L} 5$-FOA). Solid media were prepared adding $2 \%$ agar before autoclaving.

Yeast strains were freshly defrosted from stocks and grown at $30^{\circ} \mathrm{C}$, except for $s c c 1-73$ strains that were grown at $26^{\circ} \mathrm{C}$, using standard practices. All experiments were performed at $30^{\circ} \mathrm{C}$ unless specified.

Plasmids. Plasmids pCM189-L2HOr and pRS316-TINV 6 carrying the leu2-HOr allele, pWJ1344 carrying Rad52-YFP fusion ${ }^{69}$, pSCH204 carrying the $L$-LacZ direct-repeat recombination system ${ }^{70}$, pRS316, pFA6aKANMX4, pFA6aHPHMX4, and pFA6aNATNT2 used for gene replacement ${ }^{68,71}$, YEplac112, YEplac112-Rpd3 and YEplac112-H150A ${ }^{34}$, PEN149, pRS315, or PEN153 (ref. ${ }^{40}$ ) have been previously described.

pTHGH was generated by inserting the XmaI-SalI GAL-HO fragment from pRS313GAL-HO ${ }^{24}$ into pRS316-TINV ${ }^{6}$. pRS316-FRTa and pRS316-FRTb carrying the leu2-FRT allele with FRT site site in two orientations were generated by inserting the EcoRI-digested FRT sequence, obtained by primer (EcoRI-FRT-1 and EcoRI-FRT-2) annealing of FRT flanked with EcoRI restriction site sequences, into 
EcoRI-digested pRS316-LEU2. Previously, pRS316-LEU2 was obtained by cloning a BamHI-HindIII fragment from pCM189-LEU2 (refs. 6,72) into pRS316. pTINVFRT was constructed by substitution of BstEII-HindIII fragment of pTINV-HO for BstEII-HindII fragment of pRS316-FRTa. pTINV-FRTb containing the leu2-FRT allele was constructed by substitution of BstEII-HindIII fragment of pTINV-HO for BstEII-HindII fragment of pRS316-FRTb. pTINV-FRTd and pTINV-FRTg were generated by religation of $P v u I I$ digested pTINV-FRT and pTINV-FRTb, respectively.

Genotoxic damage sensitivity assay. Mid-log cultures were grown in YPAD medium. Ten-fold dilutions of the culture prepared in sterile water were plated on solid YPAD medium containing the drugs at the indicated concentrations. UV irradiation was performed in the dried plates. Plates were incubated during 3 days (in the dark for UV-irradiated plates).

Physical analysis of SCE intermediates. Cells transformed with pTHGH, pRS316-TINV, pTINVFRT, pTINV-FRTd, or pTINVFRTg were grown to mid-log phase normally in SRaf (except in Fig. 1a-c, which were grown in SGL) and at $30^{\circ}$ $\mathrm{C}$ unless otherwise specified. In all cases, $5 \mu \mathrm{g} / \mathrm{mL}$ doxycycline was used to repress transcription from the TET promoter (tetp). Then, galactose (2\%) was added to induce $\mathrm{HO}$ or FLPm expression. Samples were collected and DNA was extracted, digested with SpeI-XhoI (New England Biolabs), and analyzed by Southern blot hybridization using Hybond XL+ (GE Healthcare) membranes and detected with a ${ }^{32} \mathrm{P}$-labeled $0.22-\mathrm{kb} L E U 2$ probe $^{73}$. Original blots are provided as Source Data file. The LEU2 probe was obtained by PCR using Leu2 Up 2000 and Leu Lo 2000 primers (Supplementary Table 2) and purified from agarose gels just before use. Quantification was performed by calculating the signal of the bands corresponding to DSBs, SCE, or SCE + ICR fragments relative to the total DNA in each line. For the analysis of nicks, DNA samples were electrophoresed at $4{ }^{\circ} \mathrm{C}$ in alkaline $(50$ mM NaOH, 1 mM EDTA) conditions. PhosphorImager Fujifilm FLA-5100 and the ImageGauge program were used for quantification.

Genetic analysis of recombination. Recombination frequencies were calculated as the median value of six independent colonies. The mean value of three independent transformants was plotted. For the LlacZ system, yeasts were grown in SC-trp plates and $\mathrm{Leu}^{+}$recombinants were selected in SC-leu-trp. Recombination tests were performed with the TINV-HO or TINV-FRT systems. Briefly, mid-log phase cultures of yeast carrying the $\mathrm{HO}$ or FLPm gene under the control of the GAL1 promoter were normally grown in SRaf (except in Figs. 1d and 3a, which were grown in SGL) liquid media with $5 \mu \mathrm{g} / \mathrm{mL}$ of doxycycline and at $30^{\circ} \mathrm{C}$ unless indicated and split into two halves. One half was maintained in SRaf (or SGL in Figs. 1d and 3a) $(-\mathrm{HO},-\mathrm{FLPm})$ and $2 \%$ of galactose was added to the other half $(+\mathrm{HO},+\mathrm{FLPm})$ for $5(\mathrm{HO})$ or 6 (FLP) hours. $\mathrm{Leu}^{+}$recombinants were selected on SC-leu-ura.

Chromosomal unequal SCR. Chromosomal unequal SCR was assayed at the his $3 \Delta 5^{\prime}:$ his $3 \Delta 3^{\prime}$ system $^{30}$. In this case, yeasts were grown in YPAD plates and $\mathrm{His}^{+}$ recombinants were selected on SC-his. The mean of three independent experiments was plotted.

Double-stranded DNA gap repair. The frequency of double-stranded DNA gap repair was calculated as the number of recombinants obtained after transformation with $200 \mathrm{ng}$ of MfeI-digested pCM189-L2HOr plasmid divided by the number of transformants obtained with $200 \mathrm{ng}$ of undigested plasmid ${ }^{23}$. The mean of three independent experiments was plotted.

Molecular detection of BIR intermediates. For the detection of BIR intermediates, $2 \%$ galactose was added to induce $\mathrm{HO}$ expression to mid-log cultures of yeast strains containing the BIR assay grown in SRaf. DNA was then extracted and subjected to PCR reactions with different primers ${ }^{33}$. Original gels are provided as Source Data file. PhosphorImager Fujifilm FLA-5100 and the ImageGauge program were used for quantifying. The signal intensity of the PCR products amplified with $\mathrm{p} 1$ and $\mathrm{p} 2$ primers (Supplementary Table 2) was normalized to those of the PCR with $\mathrm{p} 1$ and $\mathrm{p} 4$ primers (Supplementary Table 2).

Plasmid loss. Colonies of independent transformants carrying the pRS316 plasmid were grown in YPAD plates for 2 days. Several dilutions were plated in YPAD (to score for total cells) and SC-trp (to score for cells which have lost the pRS316 plasmid). The frequency of plasmid loss was calculated as the median value of six independent colonies. The mean of three independent transformants was plotted.

Gross chromosomal rearrangements. Loss of CAN1 and URA 3 marker were selected by plating late-log phase culture in SC-FOA with L-cannavanine $(60 \mathrm{mg} / \mathrm{L})^{74}$. The median of three independent experiments was plotted.

Analyses of Rad52 foci. Rad52 foci were counted in more than 200 S/G2 cells

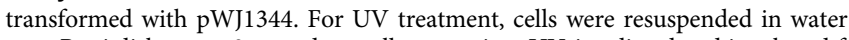
onto Petri dishes as a 3-mm-deep cell suspension, UV-irradiated and incubated for
$2 \mathrm{~h}$ before counting. For MMS treatment, MMS was added at the concentration indicated and incubated for $2 \mathrm{~h}$ before counting. Cells were visualized in Leica DC 350F. The mean and SEM of three different experiments was plotted.

Chromosome immunprecipitation. A yeast cell culture exponentially growing in SRaf media was split in two. One half was supplemented with $2 \%$ glucose and the other half with $2 \%$ galactose to induce $\mathrm{HO}$ induction $(+\mathrm{HO})$ for $2 \mathrm{~h}$. Cultures were crosslinked during $30 \mathrm{~min}$ with formaldehyde ( $1 \%$ final concetration). The reaction was stopped with glycine (125 mM final concentration). Samples were washed twice with PBS and collected into $1 \mathrm{~mL}$ tubes. Pellets were resuspended in lysis buffer (50 mM HEPES-KOH pH 7.5, $140 \mathrm{mM} \mathrm{NaCl}, 1 \mathrm{mM}$ EDTA pH 8, Triton X$100,0.1 \%$ sodium deoxycholate), complemented with protease inhibitors (cOmplete protease inhibitor cocktail (Roche) and $1 \mathrm{mM}$ PMSF). Cell lysis was performed by shaking for $45 \mathrm{~min}$ with glass beads. Samples were collected and sonicated using Bioruptor (Diagenode), with cycles of $30 \mathrm{~s}$ during $30 \mathrm{~min}$. Cell debris was eliminated by two rounds of centrifugation. Immunoprecipitation using Dynabeads Protein G (Invitrogen) for c-Myc monoclonal antibody (Clontech, cat no. 631206, 3:400) was carried out at $4^{\circ} \mathrm{C}$ overnight and samples were washed four times with different solutions. Lysis buffer, lysis buffer complemented with 500 $\mathrm{mM} \mathrm{NaCl}$, a solution containing $10 \mathrm{mM}$ Tris- $\mathrm{HCl}$ pH 8, $1 \mathrm{mM}$ EDTA pH 8, 250 $\mathrm{mM} \mathrm{LiCl}, 0.5 \%$ IGEPAL (Sigma), $0.5 \%$ SDS, and $0.5 \%$ sodium deoxycholate and last, a solution containing $10 \mathrm{mM}$ Tris-HCl, $1 \mathrm{mM}$ EDTA pH 8. Chromatin was eluted at $65^{\circ} \mathrm{C}$ for $10 \mathrm{~min}$ with $150 \mu \mathrm{L}$ of $50 \mathrm{mM}$ Tris-HCl pH 7.4, $10 \mathrm{mM}$ EDTA, $1 \% \mathrm{SDS}$, then treated with $6 \mu \mathrm{L}$ of $50 \mathrm{mg} / \mathrm{mL}$ pronase at $42^{\circ} \mathrm{C}$ for $2 \mathrm{~h}$ and then decrosslinked for $6 \mathrm{~h}$ at $65^{\circ} \mathrm{C}$. DNA was cleaned up with a Quiagen purification kit. PCR primers used are shown in Supplementary Table 2.

Sister chromatid cohesion assay. SBY885 derivative strains (provided by Sue Biggins) were grown in YPAD to mid-log phase and supplemented with $25 \mu \mathrm{M}$ $\mathrm{CuSO}_{4}$ to induce GFP expression. Then cells were G1-arrested with $2.5 \mu \mathrm{M}$ of $\alpha$ factor or G2/M-arrested with $15 \mathrm{mg} / \mathrm{mL}$ nocodazole. One milliliter of culture was fixed with $2.5 \%$ formaldehyde and visualized at the fluorescence microscope (Leica DC 350F). More than 200 cells were analyzed in each experiment.

Reporting Summary. Further information on research design is available in the Nature Research Reporting Summary linked to this article.

\section{Data Availability}

Data underlying figures are provided as Source Data files. All data supporting the findings in the manuscript are available from the corresponding authors upon reasonable request.

Received: 13 May 2019; Accepted: 22 October 2019; Published online: 15 November 2019

\section{References}

1. Gaillard, H., Garcia-Muse, T. \& Aguilera, A. Replication stress and cancer. Nat. Rev. 15, 276-289 (2015).

2. Tomasetti, C., Li, L. \& Vogelstein, B. Stem cell divisions, somatic mutations, cancer etiology, and cancer prevention. Science 355, 1330-1334 (2017).

3. Lindahl, T. \& Barnes, D. E. Repair of endogenous DNA damage. Cold Spring Harb. Symp. Quant. Biol. 65, 127-133 (2000).

4. Tubbs, A., Nussenzweig, A. \& Endogenous, D. N. A. Endogenous DNA damage as a source of genomic instability in cancer. Cell 168, 644-656 (2017).

5. Cortez, D. Preventing replication fork collapse to maintain genome integrity. DNA Repair (Amst). 32, 149-157 (2015)

6. Gonzalez-Barrera, S., Cortes-Ledesma, F., Wellinger, R. E. \& Aguilera, A. Equal sister chromatid exchange is a major mechanism of double-strand break repair in yeast. Mol. Cell 11, 1661-1671 (2003).

7. Johnson, R. D. \& Jasin, M. Sister chromatid gene conversion is a prominent double-strand break repair pathway in mammalian cells. EMBO J. 19, 3398-3407 (2000).

8. Kadyk, L. C. \& Hartwell, L. H. Sister chromatids are preferred over homologs as substrates for recombinational repair in Saccharomyces cerevisiae. Genetics 132, 387-402 (1992).

9. Pardo, B., Gomez-Gonzalez, B. \& Aguilera, A. DNA repair in mammalian cells: DNA double-strand break repair: how to fix a broken relationship. Cell. Mol. Life Sci. 66, 1039-1056 (2009).

10. Cortes-Ledesma, F. \& Aguilera, A. Double-strand breaks arising by replication through a nick are repaired by cohesin-dependent sister-chromatid exchange. EMBO Rep. 7, 919-926 (2006).

11. Sjogren, C. \& Strom, L. S-phase and DNA damage activated establishment of sister chromatid cohesion-importance for DNA repair. Exp. Cell Res. 316, 1445-1453 (2010). 
12. Kim, J. S., Krasieva, T. B., LaMorte, V., Taylor, A. M. \& Yokomori, K. Specific recruitment of human cohesin to laser-induced DNA damage. J. Biol. Chem. 277, 45149-45153 (2002).

13. Sjogren, C. \& Nasmyth, K. Sister chromatid cohesion is required for postreplicative double-strand break repair in Saccharomyces cerevisiae. Curr. Biol. 11, 991-995 (2001).

14. Strom, L., Lindroos, H. B., Shirahige, K. \& Sjogren, C. Postreplicative recruitment of cohesin to double-strand breaks is required for DNA repair. Mol. Cell 16, 1003-1015 (2004).

15. Unal, E. et al. DNA damage response pathway uses histone modification to assemble a double-strand break-specific cohesin domain. Mol. Cell 16, 991-1002 (2004)

16. Strom, L. et al. Postreplicative formation of cohesion is required for repair and induced by a single DNA break. Science 317, 242-245 (2007).

17. Unal, E., Heidinger-Pauli, J. M. \& Koshland, D. DNA double-strand breaks trigger genome-wide sister-chromatid cohesion through Ecol (Ctf7). Science 317, 245-248 (2007).

18. De Piccoli, G. et al. Smc5-Smc6 mediate DNA double-strand-break repair by promoting sister-chromatid recombination. Nat. Cell Biol. 8, 1032-1034 (2006).

19. Potts, P. R., Porteus, M. H. \& Yu, H. Human SMC5/6 complex promotes sister chromatid homologous recombination by recruiting the $\mathrm{SMC} 1 / 3$ cohesin complex to double-strand breaks. EMBO J. 25, 3377-3388 (2006)

20. Conde, F. et al. The Dot1 histone methyltransferase and the Rad9 checkpoint adaptor contribute to cohesin-dependent double-strand break repair by sister chromatid recombination in Saccharomyces cerevisiae. Genetics 182, 437-446 (2009). 109.101899.

21. Oum, J. H. et al. RSC facilitates Rad59-dependent homologous recombination between sister chromatids by promoting cohesin loading at DNA doublestrand breaks. Mol. Cell. Biol. 31, 3924-3937 (2011).

22. Cortes-Ledesma, F., Tous, C. \& Aguilera, A. Different genetic requirements for repair of replication-born double-strand breaks by sister-chromatid recombination and break-induced replication. Nucleic Acids Res. 35, 6560-6570 (2007).

23. Munoz-Galvan, S. et al. A new role for Rrm3 in repair of replication-born DNA breakage by sister chromatid recombination. PLoS Genet. 13, e1006781 (2017).

24. Munoz-Galvan, S. et al. Competing roles of DNA end resection and nonhomologous end joining functions in the repair of replication-born doublestrand breaks by sister-chromatid recombination. Nucleic Acids Res. 41, 1669-1683 (2013).

25. Masumoto, H., Hawke, D., Kobayashi, R. \& Verreault, A. A role for cell-cycleregulated histone $\mathrm{H} 3$ lysine 56 acetylation in the DNA damage response. Nature 436, 294-298 (2005).

26. Munoz-Galvan, S., Jimeno, S., Rothstein, R. \& Aguilera, A. Histone H3K56 acetylation, Rad52, and non-DNA repair factors control double-strand break repair choice with the sister chromatid. PLoS Genet. 9, e1003237 (2013).

27. Qian, W., Ma, D., Xiao, C., Wang, Z. \& Zhang, J. The genomic landscape and evolutionary resolution of antagonistic pleiotropy in yeast. Cell Rep. 2, 1399-1410 (2012).

28. Carrozza, M. J. et al. Histone H3 methylation by Set2 directs deacetylation of coding regions by Rpd3S to suppress spurious intragenic transcription. Cell 123, 581-592 (2005)

29. Morillo-Huesca, M., Clemente-Ruiz, M., Andujar, E. \& Prado, F. The SWR1 histone replacement complex causes genetic instability and genome-wide transcription misregulation in the absence of H2A.Z. PLoS One 5, e12143 (2010).

30. Fasullo, M. T. \& Davis, R. W. Recombinational substrates designed to study recombination between unique and repetitive sequences in vivo. Proc. Natl. Acad. Sci. USA 84, 6215-6219 (1987).

31. Moriel-Carretero, M. \& Aguilera, A. A postincision-deficient TFIIH causes replication fork breakage and uncovers alternative Rad51- or Pol32-mediated restart mechanisms. Mol. Cell 37, 690-701 (2010).

32. Rundlett, S. E. et al. HDA1 and RPD3 are members of distinct yeast histone deacetylase complexes that regulate silencing and transcription. Proc. Natl. Acad. Sci. USA 93, 14503-14508 (1996).

33. Ruiz, J. F., Gomez-Gonzalez, B. \& Aguilera, A. Chromosomal translocations caused by either pol32-dependent or pol32-independent triparental breakinduced replication. Mol. Cell. Biol. 29, 5441-5454 (2009).

34. Kadosh, D. \& Struhl, K. Histone deacetylase activity of Rpd3 is important for transcriptional repression in vivo. Genes Dev. 12, 797-805 (1998).

35. Nielsen, I. et al. A Flp-nick system to study repair of a single protein-bound nick in vivo. Nat. Methods 6, 753-757 (2009).

36. Mayle, R. et al. DNA REPAIR. Mus81 and converging forks limit the mutagenicity of replication fork breakage. Science 349, 742-747 (2015).

37. Michaelis, C., Ciosk, R. \& Nasmyth, K. Cohesins: chromosomal proteins that prevent premature separation of sister chromatids. Cell 91, 35-45 (1997).

38. Blat, Y. \& Kleckner, N. Cohesins bind to preferential sites along yeast chromosome III, with differential regulation along arms versus the centric region. Cell 98, 249-259 (1999).
39. Thaminy, S. et al. Hst3 is regulated by Mec1-dependent proteolysis and controls the $\mathrm{S}$ phase checkpoint and sister chromatid cohesion by deacetylating histone H3 at lysine 56. J. Biol. Chem. 282, 37805-37814 (2007).

40. Ruiz-Roig, C., Vieitez, C., Posas, F. \& de Nadal, E. The Rpd3L HDAC complex is essential for the heat stress response in yeast. Mol. Microbiol. 76, 1049-1062 (2010).

41. Borges, V. et al. Hos1 deacetylates Smc3 to close the cohesin acetylation cycle. Mol. Cell 39, 677-688 (2010).

42. Beckouet, F. et al. An Smc3 acetylation cycle is essential for establishment of sister chromatid cohesion. Mol. Cell 39, 689-699 (2010).

43. Tamburini, B. A. \& Tyler, J. K. Localized histone acetylation and deacetylation triggered by the homologous recombination pathway of double-strand DNA repair. Mol. Cell. Biol. 25, 4903-4913 (2005).

44. Hakimi, M. A. et al. A chromatin remodelling complex that loads cohesin onto human chromosomes. Nature 418, 994-998 (2002).

45. Baetz, K. K., Krogan, N. J., Emili, A., Greenblatt, J. \& Hieter, P. The ctf13-30/ CTF13 genomic haploinsufficiency modifier screen identifies the yeast chromatin remodeling complex RSC, which is required for the establishment of sister chromatid cohesion. Mol. Cell. Biol. 24, 1232-1244 (2004).

46. Huang, J., Hsu, J. M. \& Laurent, B. C. The RSC nucleosome-remodeling complex is required for Cohesin's association with chromosome arms. Mol. Cell 13, 739-750 (2004).

47. Riedel, C. G., Gregan, J., Gruber, S. \& Nasmyth, K. Is chromatin remodeling required to build sister-chromatid cohesion? Trends Biochem. Sci. 29, 389-392 (2004).

48. Bernard, P. et al. Requirement of heterochromatin for cohesion at centromeres. Science 294, 2539-2542 (2001).

49. Nonaka, N. et al. Recruitment of cohesin to heterochromatic regions by Swi6/ HP1 in fission yeast. Nat. Cell Biol. 4, 89-93 (2002).

50. Partridge, J. F., Scott, K. S., Bannister, A. J., Kouzarides, T. \& Allshire, R. cisacting DNA from fission yeast centromeres mediates histone $\mathrm{H} 3$ methylation and recruitment of silencing factors and cohesin to an ectopic site. Curr. Biol. 12, 1652-1660 (2002)

51. Chang, C. R., Wu, C. S., Hom, Y. \& Gartenberg, M. R. Targeting of cohesin by transcriptionally silent chromatin. Genes Dev. 19, 3031-3042 (2005).

52. Wu, C. S., Chen, Y. F. \& Gartenberg, M. R. Targeted sister chromatid cohesion by Sir2. PLoS Genet. 7, e1002000 (2011).

53. Tittel-Elmer, M. et al. Cohesin association to replication sites depends on rad50 and promotes fork restart. Mol. Cell 48, 98-108 (2012).

54. Frattini, C. et al. Cohesin ubiquitylation and mobilization facilitate stalled replication fork dynamics. Mol. Cell 68, 758-772 e754 (2017).

55. Gomez-Gonzalez, B., Patel, H., Early, A. \& Diffley, J. F. X. Rpd3L contributes to the DNA damage sensitivity of Saccharomyces cerevisiae checkpoint mutants. Genetics 211, 503-513 (2019).

56. Salas-Armenteros, I. et al. Human THO-Sin $3 \mathrm{~A}$ interaction reveals new mechanisms to prevent R-loops that cause genome instability. EMBO J. 36, 3532-3547 (2017)

57. Tang, J. et al. Acetylation limits 53BP1 association with damaged chromatin to promote homologous recombination. Nat. Struct. Mol. Biol. 20, 317-325 (2013).

58. Atienza, J. M. et al. Suppression of RAD21 gene expression decreases cell growth and enhances cytotoxicity of etoposide and bleomycin in human breast cancer cells. Mol. Cancer Ther. 4, 361-368 (2005).

59. Schmitz, J., Watrin, E., Lenart, P., Mechtler, K. \& Peters, J. M. Sororin is required for stable binding of cohesin to chromatin and for sister chromatid cohesion in interphase. Curr. Biol. 17, 630-636 (2007).

60. Sonoda, E. et al. Rad51-deficient vertebrate cells accumulate chromosomal breaks prior to cell death. EMBO J. 17, 598-608 (1998).

61. Gelot, C. et al. The cohesin complex prevents the end joining of distant DNA double-strand ends. Mol. Cell 61, 15-26 (2016).

62. Hill, V. K., Kim, J. S. \& Waldman, T. Cohesin mutations in human cancer. Biochim. Biophys. Acta 1866, 1-11 (2016).

63. Parenti, I. et al. Mutations in chromatin regulators functionally link Cornelia de Lange syndrome and clinically overlapping phenotypes. Hum. Genet. 136, 307-320 (2017).

64. Gordillo, M. et al. The molecular mechanism underlying Roberts syndrome involves loss of ESCO2 acetyltransferase activity. Hum. Mol. Genet. 17, 2172-2180 (2008).

65. Liu, J. \& Krantz, I. D. Cornelia de Lange syndrome, cohesin, and beyond. Clin. Genet. 76, 303-314 (2009).

66. Vrouwe, M. G. et al. Increased DNA damage sensitivity of Cornelia de Lange syndrome cells: evidence for impaired recombinational repair. Hum. Mol. Genet. 16, 1478-1487 (2007).

67. Tsalik, E. L. \& Gartenberg, M. R. Curing Saccharomyces cerevisiae of the 2 micron plasmid by targeted DNA damage. Yeast 14, 847-852 (1998).

68. Wach, A., Brachat, A., Pohlmann, R. \& Philippsen, P. New heterologous modules for classical or PCR-based gene disruptions in Saccharomyces cerevisiae. Yeast 10, 1793-1808 (1994). 
69. Lisby, M., Rothstein, R. \& Mortensen, U. H. Rad52 forms DNA repair and recombination centers during $\mathrm{S}$ phase. Proc. Natl. Acad. Sci. USA 98, 8276-8282 (2001).

70. Chavez, S. \& Aguilera, A. The yeast HPR1 gene has a functional role in transcriptional elongation that uncovers a novel source of genome instability. Genes Dev. 11, 3459-3470 (1997).

71. Janke, C. et al. A versatile toolbox for PCR-based tagging of yeast genes: new fluorescent proteins, more markers and promoter substitution cassettes. Yeast 21, 947-962 (2004)

72. Gonzalez-Barrera, S., Garcia-Rubio, M. \& Aguilera, A. Transcription and double-strand breaks induce similar mitotic recombination events in Saccharomyces cerevisiae. Genetics 162, 603-614 (2002).

73. Gomez-Gonzalez, B., Ruiz, J. F. \& Aguilera, A. Genetic and molecular analysis of mitotic recombination in Saccharomyces cerevisiae. Methods Mol. Biol. (Clifton, N. J.) 745, 151-172 (2011).

74. Schmidt, K. H., Pennaneach, V., Putnam, C. D. \& Kolodner, R. D. Analysis of gross-chromosomal rearrangements in Saccharomyces cerevisiae. Methods Enzymol. 409, 462-476 (2006).

\section{Acknowledgements}

We thank Sue Biggins, Marc Gartenberg, Eulalia de Nadal, Kevin Struhl, and Jordi TorresRosell for kindly providing plasmids and strains. Research was supported by the European Research Council (ERC2014 AdG669898 TARLOOP), the Spanish Ministry of Economy and Competitiveness (BFU2016-75058-P), and the European Union (FEDER). P.O. was supported by a predoctoral training grant from the Spanish Ministry of Economy and Competitiveness and B.G.-G. by the Spanish Association Against Cancer (AECC).

\section{Author contributions}

P.O. performed the experiments; P.O., B.G.-G. and A.A. designed the experiments and wrote the manuscript. All authors read, discussed, and agreed with the final version of this manuscript.

\section{Competing interests}

The authors declare no competing interests.

\section{Additional information}

Supplementary information is available for this paper at https://doi.org/10.1038/s41467019-13210-5.

Correspondence and requests for materials should be addressed to B.Góm-G. or A.A.

Peer review information Nature Communications thanks Jennifer Cobb and the other, anonymous, reviewer(s) for their contribution to the peer review of this work. Peer reviewer reports are available.

Reprints and permission information is available at http://www.nature.com/reprints

Publisher's note Springer Nature remains neutral with regard to jurisdictional claims in published maps and institutional affiliations.

(c) (i) Open Access This article is licensed under a Creative Commons Attribution 4.0 International License, which permits use, sharing, adaptation, distribution and reproduction in any medium or format, as long as you give appropriate credit to the original author(s) and the source, provide a link to the Creative Commons license, and indicate if changes were made. The images or other third party material in this article are included in the article's Creative Commons license, unless indicated otherwise in a credit line to the material. If material is not included in the article's Creative Commons license and your intended use is not permitted by statutory regulation or exceeds the permitted use, you will need to obtain permission directly from the copyright holder. To view a copy of this license, visit http://creativecommons.org/ licenses/by/4.0/.

(C) The Author(s) 2019 